\title{
ENERGY SCALING LAW FOR A SINGLE DISCLINATION IN A THIN ELASTIC SHEET
}

\author{
HEINER OLBERMANN
}

\begin{abstract}
We consider a single disclination in a thin elastic sheet of thickness $h$. We prove ansatz-free lower bounds for the free elastic energy in three different settings: First, for a geometrically fully non-linear plate model, second, for three-dimensional nonlinear elasticity, and third, for the Föppl-von Kármán plate theory. The lower bounds in the first and third result are optimal in the sense that we find upper bounds that are identical to the respective lower bounds in the leading order of $h$.
\end{abstract}

\section{INTRODUCTION}

1.1. Setup and previous work. Consider the following setup: Take a thin elastic sheet in the shape of a disc, and remove a sector from it. Then glue the edges of the cut back together. The (stress free) reference metric of the sheet is now that of a singular cone. In other words, the reference metric is flat away from the centre, where the Gauss curvature has a $\delta$-type singularity. In the literature, such a defect of the reference metric at the origin is often called a "disclination". We are interested in upper and lower bounds for the free elastic energy of the sheet with a single disclination. Note that we we do not impose boundary conditions or other constraints.

Disclinations play an important role, e.g., in the modeling of cell walls [29, 18], where they can serve as the vertices in topologically spherical shells, that take on the shape of a (smoothed) icosahedron. Such a behaviour has been observed, e.g., for virus capsids [4]. Recent interest in disclinations in thin sheets comes from the analysis of carbon nanofilms [28] and the modeling of biological growth processes [9]. An extensive review of the role of disclinations in the mechanics of solids, not limited to the case of thin sheets, can be found in [28].

Considering a single disclination of a comparable deficit without boundary conditions, one expects that energy will be minimized by a shape that looks like a cone away from the disclination, and deviates from the cone on a ball around the disclination whose size is comparable to the thickness of the sheet. In the literature, energy estimates for a single disclination have so far been ansatz based (as in [29, 18, 35]), or it has been assumed that the deformation is radially symmetric. In the latter case, the dimensionality of the problem is reduced from two to one, and ODE methods can be used. It has been shown in 23] that under this assumption, in the Föppl-von Kármán approximation, minimizers do indeed show the behavior described above. Additionally, this paper contains a quantitative estimate on how fast minimizers approach the singular cone as the distance $r$ from the apex of the cone increases.

Date: October 14, 2018. 
In the recent work 26, we suggested an ansatz how to prove lower bounds for the free elastic energy of a single disclination without the assumption of radial symmetry. We viewed the elastic sheet in the deformed configuration as an immersed Riemannian manifold, and focused our analysis on intrinsically defined objects, such as the metric and Gauss curvature of the manifold. The main idea brought forward in this work was that estimates for integrals of Gauss curvature can be obtained by interpolation between the metric and the Gauss curvature. Lower bounds for integrals of Gauss curvature can be translated to lower bounds on the bending energy by the isoperimetric inequality on the sphere. However, in [26], we had to modify the elastic energy and make simplifying assumptions on the shape of the sheet to put this ansatz to work.

Here, we report on an improvement of the method of proof, that makes the simplifying assumptions unnecessary altogether. One further benefit of our modified approach is that it makes the use of the jargon of Riemannian geometry superfluous.

1.2. Statement of results. Our first result regards a single disclination in a geometrically fully nonlinear plate model. Let $B_{1}:=\left\{x \in \mathbb{R}^{2}:|x|<1\right\}$ be the sheet in the reference configuration. The singular cone may be described by the mapping $y^{\Delta}: B_{1} \rightarrow \mathbb{R}^{3}$,

$$
y^{\Delta}(x)=\sqrt{1-\Delta^{2}} x+\Delta|x| e_{3} .
$$

Here, $0<\Delta<1$ is the height of the singular cone, and is determined by the deficit of the disclination at the origin. The reference metric on $B_{1}$ is given by

$$
\begin{aligned}
g_{\Delta}(x) & =D y^{\Delta}(x)^{T} D y^{\Delta}(x) \\
& =\left(1-\Delta^{2}\right) \hat{x} \otimes \hat{x}+\Delta^{2} \hat{x} \otimes \hat{x} \\
& =\operatorname{Id}_{2 \times 2}-\Delta^{2} \hat{x}^{\perp} \otimes \hat{x}^{\perp},
\end{aligned}
$$

where $\hat{x}=x /|x|$ and $\hat{x}^{\perp}=\left(-x_{2}, x_{1}\right) /|x|$. The induced metric of a deformation $y \in$ $W^{2,2}\left(B_{1} ; \mathbb{R}^{3}\right)$ is

$$
g_{y}=D y^{T} D y \text {. }
$$

The free elastic energy $I_{h, \Delta}: W^{2,2}\left(B_{1} ; \mathbb{R}^{3}\right) \rightarrow \mathbb{R}$ is defined by

$$
I_{h, \Delta}(y)=\int_{B_{1}}\left(\left|g_{y}-g_{\Delta}\right|^{2}+h^{2}\left|D^{2} y\right|^{2}\right) \mathrm{d} \mathcal{L}^{2},
$$

where $\mathrm{d} \mathcal{L}^{2}$ denotes 2-dimensional Lebesgue measure. The first result of the present paper is

Theorem 1. There exists a constant $C=C(\Delta)$ with the following property:

$$
2 \pi \Delta^{2} h^{2}\left(|\log h|-\frac{3}{2} \log |\log h|-C\right) \leq \min _{y \in W^{2,2}\left(B_{1} ; \mathbb{R}^{3}\right)} I_{h, \Delta}(y) \leq 2 \pi \Delta^{2} h^{2}(|\log h|+C)
$$

for all small enough $h$.

Before we come to the statement of the other theorems, let us try to explain the improvement of our techniques that allows us to drop the additional assumptions we had to make in [26]. It consists in noting that the function $\sum_{i=1}^{3} \operatorname{det} D^{2} y_{i}$ is controlled by both the membrane and the bending term, in different functions spaces. Indeed, it is not difficult 
to see that $\sum_{i=1}^{3} \operatorname{det} D^{2} y_{i}$ is bounded in $L^{1}$ by the bending energy. The control exerted by the membrane energy is slightly more subtle. We recall the very weak form of the Hessian:

$$
\operatorname{det} D^{2} w=\left(w, 1, w_{, 2}\right)_{, 12}-\frac{1}{2}\left(\left|w_{, 1}\right|^{2}\right)_{, 22}-\frac{1}{2}\left(\left|w_{, 2}\right|^{2}\right)_{, 11} \quad \text { for } w \in C^{2} .
$$

Hence,

$$
\sum_{i=1}^{3} \operatorname{det} D^{2} y_{i}=\left(y_{, 1} \cdot y_{, 2}\right)_{, 12}-\frac{1}{2}\left(\left|y_{, 1}\right|^{2}\right)_{, 22}-\frac{1}{2}\left(\left|y_{, 2}\right|^{2}\right)_{, 11} \quad \text { for } y \in C^{2}\left(B_{1} ; \mathbb{R}^{3}\right),
$$

and we see that $\sum_{i=1}^{3} \operatorname{det} D^{2} y_{i}$ is controlled by the membrane term in $W^{-2,1}$. Thus, by interpolation, we can get control over integrals of $\sum_{i=1}^{3} \operatorname{det} D^{2} y_{i}$ in $L^{1}$. By the Sobolev inequality in $\mathbb{R}^{2}$, lower bounds for these integrals can be translated to lower bounds for $\left|D^{2} y\right|$ (see Section 2), and hence for the bending energy.

As a consequence of Theorem 1, we will be able to prove a scaling law in three-dimensional elasticity. Let arccos : $[-1,1] \rightarrow[0, \pi]$ denote the inverse of $\cos :[0, \pi] \rightarrow[-1,1]$. Define

$$
B_{1, \Delta}:=\left\{x=\left(x_{1}, x_{2}\right) \in B_{1} \backslash\{0\}: 0 \leq \arccos \frac{x_{1}}{|x|}<\sqrt{1-\Delta^{2}} \pi\right\} .
$$

Let $\mathbb{R}_{-}:=\left\{\left(x_{1}, 0\right): x_{1} \leq 0\right\}$, and let $\varphi: \mathbb{R}^{2} \backslash \mathbb{R}_{-} \rightarrow \mathbb{R}$ be the angular coordinate satisfying $x=|x|(\cos \varphi(x), \sin \varphi(x))$ with values in $(-\pi, \pi)$. We define the map $\iota_{\Delta}: \mathbb{R}^{2} \backslash \mathbb{R}_{-} \rightarrow B_{1}$ by

$$
\iota_{\Delta}(x)=\left(|x| \cos \frac{\varphi(x)}{\sqrt{1-\Delta^{2}}},|x| \sin \frac{\varphi(x)}{\sqrt{1-\Delta^{2}}}\right) .
$$

Now let $\iota_{\Delta}^{(3)}: B_{1, \Delta} \times[-h / 2, h / 2] \rightarrow \mathbb{R}^{2} \times[-h / 2, h / 2]$ be defined by

$$
\iota_{\Delta}^{(3)}\left(x^{\prime}, x_{3}\right)=\left(\iota_{\Delta}\left(x^{\prime}\right), x_{3}\right) .
$$

Note that $\left(\iota_{\Delta}^{(3)}\right)^{-1}$ is defined almost everywhere on $B_{1} \times[-h / 2, h / 2]$. We define the set of allowed deformations to be

$$
\begin{aligned}
& \mathcal{A}_{h, \Delta}:=\left\{Y \in W^{1,2}\left(B_{1, \Delta} \times[-h / 2, h / 2] ; \mathbb{R}^{3}\right)\right. \\
&\text { such that } \left.Y \circ\left(\iota_{\Delta}^{(3)}\right)^{-1} \in W^{1,2}\left(B_{1} \times[-h / 2, h / 2] ; \mathbb{R}^{3}\right)\right\} .
\end{aligned}
$$

Let $W \in C\left(\mathbb{R}^{3 \times 3} ;[0, \infty)\right)$ be the stored energy density that is $S O(3)$-invariant, i.e.,

$$
W(R F)=W(F) \quad \text { for all } R \in S O(3), F \in \mathbb{R}^{3 \times 3},
$$

and such that it vanishes on $S O(3)$ and has quadratic growth, i.e.,

$$
W(F) \geq C \operatorname{dist}^{2}(F, S O(3)) \quad \text { for all } F \in \mathbb{R}^{3 \times 3},
$$

and

$$
W(F)<C_{2} \operatorname{dist}^{2}(F, S O(3)) \quad \text { for } F \in \mathcal{F}_{0},
$$

where $\mathcal{F}_{0}$ is some neighborhood of $S O(3)$ in $\mathbb{R}^{3 \times 3}$.

The energy functional is given by $E_{h, \Delta}: \mathcal{A}_{h, \Delta} \rightarrow \mathbb{R}$,

We will prove

$$
E_{h, \Delta}(Y)=\frac{1}{h} \int_{B_{1, \Delta} \times[-h / 2, h / 2]} W(D Y) \mathrm{d} x .
$$


Theorem 2. There exists a constant $C=C(\Delta, W)>0$ such that

$$
\frac{1}{C} h^{2}|\log h| \leq \min _{y \in \mathcal{A}_{h, \Delta}} E_{h, \Delta}(y) \leq C h^{2}|\log h|
$$

for all small enough $h$.

The third theorem is the analogous result to Theorem 1 in the Föppl-von Kármán setting. Here, the free elastic energy $I_{h, \Delta}^{\mathrm{vK}}$ of the deflection $(u, v) \in W^{1,2}\left(B_{1} ; \mathbb{R}^{2}\right) \times W^{2,2}\left(B_{1}\right)$ is given by

$$
I_{h, \Delta}^{\mathrm{vK}}(u, v)=\int\left|2 \operatorname{sym} D u+D v \otimes D v+\Delta^{2} \hat{x}^{\perp} \otimes \hat{x}^{\perp}\right|^{2}+h^{2}\left|D^{2} v\right|^{2} \mathrm{~d} x .
$$

Again, the parameter $\Delta$ determines the size of the deficit of the disclination. For a heuristic derivation of $I_{h, \Delta}^{\mathrm{vK}}$ from $I_{h, \Delta}$ see Section 5. Our third result is

Theorem 3. There exists a constant $C=C(\Delta)>0$ with the following property:

$$
2 \pi \Delta^{2} h^{2}(|\log h|-2 \log |\log h|-C) \leq \min _{(u, v) \in \mathcal{A}} I_{h, \Delta}^{\mathrm{vK}}(u, v) \leq 2 \pi \Delta^{2} h^{2}(|\log h|+C)
$$

for all small enough $h$.

1.3. Scientific context. In the last two and a half decades or so, there has been a lot of interest in the energy focusing in thin elastic sheets, predominantly in the physics and engineering community. The structures under consideration are typically sharp folds, conical singularities, or a (possibly very complex) combination of both. The first analysis of the scaling law for the free elastic energy in the sense of an asymptotic expansion for a thin elastic shell displaying sharp folds can be found in [34. The so-called "minimal ridge" has been analyzed in [20], see also [10, 21, 15, 19]. Structures with stress focusing in vertices (the so-called $d$-cones) were first investigated in [2, see also [5, 6, 7]. To the interested reader, we recommend the overview article by Witten [33], where many more references can be found.

In the mathematical literature, the most closely related articles to the present work are [8, 3, 24]. In [8], it has been proved that the elastic energy per unit thickness of a "single fold" scales with $h^{5 / 3}$, building on results from [31]. In [24, 3], the following has been proved: Take an elastic sheet in the shape of a disc, and prescribe the deformation of the sheet at the center and at the boundary, such that it coincides with a (singular) conical deformation there. In this class of deformations, the elastic energy per unit thickness scales with $h^{2}|\log h|$.

On a technical level, the works [8, 3, 24, treat variational problems for the functional (1), where the reference metric $g_{\Delta}$ is replaced by the flat 2-dimensional Euclidean metric, with certain Dirichlet boundary conditions. These boundary conditions are of a very special type: They are chosen such that there exists a unique Lipschitz continuous isometric immersion 1 that satisfies them, which possesses infinite bending energy. In the sequel, we will call this Lipschitz continuous isometric immersion the "singular reference configuration". Also, we will call boundary conditions "tensile", if a singular reference configuration

\footnotetext{
${ }^{1}$ By a Lipschitz continuous isometric immersion, we mean a Lipschitz map $\bar{y}: B_{1} \rightarrow \mathbb{R}^{3}$ with the property that $D \bar{y}^{T} D \bar{y}$ equals the reference metric almost everywhere.
} 
exists. The idea of proof in $[8,3,24]$ is that deviations from the singular reference configuration are penalized by the membrane energy. The optimal balance between membrane and bending energy yields the lower bound in the elastic energy.

In the setting of the single disclination, there are no tensile boundary conditions - there are none at all.

To the best of our knowledge, the method of proof using tensile boundary conditions has so far been the only one for proving ansatz-free lower bounds in variational models for thin elastic sheets with energy focusing in folds and vertices (not counting the predecessor [26] of the current work). We expect that the method of proof we present here will have applications in variational models that are sufficiently closely related to the single disclination. An interesting open question is whether or not our techniques can also be applied in settings with a flat reference metric.

1.4. $h$-principle and rigidity. We want to put Theorem 1 in yet another mathematical context. Recall the results by Nash [25] and Kuiper [16], stating that any strictly short immersion $y: M \rightarrow \mathbb{R}^{n+1}$ of an $n$-dimensional Riemannian manifold $(M, g)$ can be arbitrarily well approximated by a $C^{1}$ isometric immersion $\tilde{y}$ in the $C^{0}$ norm. I.e., given $\varepsilon>0$ and an immersion $y \in C^{1}\left(M ; \mathbb{R}^{n+1}\right)$ with $D y^{T} D y<g$ everywhere, there exists $\tilde{y} \in C^{1}\left(M ; \mathbb{R}^{n+1}\right)$ with $D \tilde{y}^{T} D \tilde{y}=g$ and $\|y-\tilde{y}\|_{C^{0}}<\varepsilon$. These results are relevant for the present context, since the leading term in the elastic energy (1), the membrane term, penalizes deviations from isometric immersions. By the Nash-Kuiper Theorem, there exists a huge amount of mutually vastly different shapes that have arbitrarily small membrane energy. In the present setting, one needs to show that all of these many degrees of freedom carry large bending energy.

Next, recall the well known Weyl problem from differential geometry: Given a Riemannian metric $g$ with positive Gauss curvature on the two-sphere $S^{2}$, find an isometric immersion $y: S^{2} \rightarrow \mathbb{R}^{3}$. Note that by the Nash-Kuiper result above, there exist many solutions if we only require $y \in C^{1}$. On the other hand, by a result by Pogorelov [27, the solution to the Weyl problem is unique (up to Euclidean motions) in the class of immersions with bounded extrinsic curvature. This class is defined by requiring that the pull-back of the volume form on $S^{2}$ under the Gauss map defines a signed Radon measure. For smooth maps, the total variation of that measure is just the $L^{1}$-norm of the Gauss curvature. We see that control over curvature "eliminates" the Nash-Kuiper constructions.

On a heuristic level, our approach can be viewed as a quantitative version of Pogorelov's rigidity result. In order to see how, note that the different results can be formulated as a statement on the measure

$$
k_{y}: U \mapsto \int_{U} K_{y} \mathrm{~d} A_{y},
$$

defined on open sets $U$, where $K_{y} \mathrm{~d} A_{y}$ is a certain curvature form (in Pogorelov's work and [26], the Gauss curvature form; for the geometrically fully nonlinear model treated here, $\sum_{i=1}^{3} \operatorname{det} D^{2} y_{i} \mathrm{~d} x$; for the Föppl-von Kármán case, $\operatorname{det} D^{2} v \mathrm{~d} x$, which is the Föpplvon Kármán version of Gauss curvature). The crucial part of Pogorelov's result is that the measure $k_{y}$ is identical to the pull back of the volume form on $S^{2}$ under the Gauss 
map if the latter is a Radon measure. Here, we give certain quantitative estimates for the difference $k_{y}-k_{0}$, where $k_{0}$ is the curvature measure associated to the reference configuration. These estimates allow for the construction of a lower bound of the bending energy via a suitable Sobolev type inequality.

By imposing tensile boundary conditions as in [8, 3, 24, one eliminates strictly short maps from the set of allowed configurations. In this way, the problem described above is circumvented. Here, we describe for the first time a setting where the constructions in the style of Nash-Kuiper are allowed by the boundary data, but shown to be energetically unfavorable.

1.5. Notation and plan of the paper. The canonical basis vectors of $\mathbb{R}^{n}$ will be denoted by $e_{1}, \ldots, e_{n}$. For a vector $x \in \mathbb{R}^{n}$, we denote its components by $x_{1}, \ldots, x_{n}$. For a vector $v=\left(v_{1}, v_{2}\right) \in \mathbb{R}^{2}$, let $v^{\perp}:=\left(-v_{2}, v_{1}\right)$. Open balls in $\mathbb{R}^{2}$ are denoted by $B_{r}:=\left\{x \in \mathbb{R}^{2}\right.$ : $|x|<r\}$. The set of linear isometries from $\mathbb{R}^{2}$ to $\mathbb{R}^{3}$ is denoted by $O(2,3)$.

For a function $f$ on $\mathbb{R}^{2}$, we use the notation $f_{, i}=\partial_{x_{i}} f$ for the partial derivatives.

The volume of the unit ball in $\mathbb{R}^{n}$ will be denoted by $\omega_{n}:=\pi^{n / 2} / \Gamma(n / 2+1)$. The symbol $\mathcal{L}^{n}$ denotes $n$-dimensional Lebesgue measure, and $\mathcal{H}^{k}$ is the $k$-dimensional Hausdorff measure. The symbol $C$ denotes numerical constants that only depend on $\Delta$. A statement such as " $f \leq C g$ " is to be understood as "there exists a constant $C=C(\Delta)>0$ such that $f \leq C g$ ". The value of $C$ may vary from one line to the next. By $g(h)=o(f(h))$ and $\tilde{g}(h)=O(\tilde{f}(h))$, we mean that

$$
\limsup _{h \rightarrow 0} \frac{|g(h)|}{|f(h)|}=0, \quad \limsup _{h \rightarrow 0} \frac{|\tilde{g}(h)|}{|\tilde{f}(h)|}<\infty
$$

respectively.

This paper is structured as follows: In Section 2, we state a Sobolev type inequality for the Brouwer degree, which will help us to translate control over $k_{y}$ into a lower bound for the bending energy later on. Theorems 1, 2 and 3 are proved in Sections 3 , 4 and 5 respectively.

\section{A Sobolev type inequality for the Brouwer Degree}

In the proof of Theorems 1 and 3 below, we will use the Sobolev inequality to get a lower bound on the bending term. From smallness of the membrane and bending energies, we will have for most $r \in[0,1]$, a lower bound on $\sum_{i=1}^{3} \mathcal{H}^{2}\left(D y_{i}\left(B_{r}\right)\right)$ in the geometrically fully nonlinear model, and on $\mathcal{H}^{2}\left(D v\left(B_{r}\right)\right)$ in the Föppl-von Kármán model (see Propositions 1 and 4). The Sobolev inequality will give us then a lower bound for $\left|D^{2} y\right|$ (in the first case) and $\left|D^{2} v\right|$ (in the second) on $\partial B_{r}$.

In the proof, we are going to need some standard facts concerning the Brouwer degree. For a more thorough exposition including proofs of the claims made here, see e.g. 11.

Let $U \subset \mathbb{R}^{n}$ be bounded, $f \in C^{\infty}\left(\bar{U} ; \mathbb{R}^{n}\right)$, and $y \in \mathbb{R}^{n} \backslash f(\partial U)$. Let $A_{y, f}$ denote the connected component of $\mathbb{R}^{n} \backslash f(\partial U)$ that contains $y$, and let $\mu$ be a smooth $n$-form on $\mathbb{R}^{n}$ with support in $A_{y, f}$ and $\int_{\mathbb{R}^{n}} \mu=1$. Then the Brouwer degree $\operatorname{deg}(y, U, f)$ is defined by

$$
\operatorname{deg}(y, U, f)=\int_{U} f^{*} \mu,
$$


where $f^{*}$ denotes the pull-back under $f$. It can be shown that this definition is independent of the choice of $\mu$, and that $\operatorname{deg}(\cdot, U, f)$ is integer-valued, and constant on connected components of $\mathbb{R}^{n} \backslash f(\partial U)$. By approximation with smooth functions, it can be shown that the Brouwer degree has a well defined meaning also for $f \in C^{0}\left(\bar{U} ; \mathbb{R}^{n}\right)$ and $y \in \mathbb{R}^{n} \backslash f(\partial U)$. From now on, let us suppose that $U \subset \mathbb{R}^{n}$ is bounded and open with Lipschitz boundary. For $f \in C^{1}\left(\bar{U} ; \mathbb{R}^{n}\right)$, and an $n$-form $\mu$ with coefficients in $L^{\infty}\left(\mathbb{R}^{n}\right)$, we have

$$
\int_{\mathbb{R}^{n}} \operatorname{deg}(y, U, f) \mu=\int_{U} f^{*} \mu .
$$

This follows immediately from the defining relation for the Brouwer degree by approximation with smooth functions. If $\mu$ is an exact form, i.e., $\mu=\mathrm{d} \omega$ for some $n-1$ form $\omega$ with coefficients in $W^{1, \infty}$, then

$$
\begin{aligned}
\int_{\mathbb{R}^{n}} \operatorname{deg}(\cdot, U, f) \mu & =\int_{U} f^{*}(\mathrm{~d} \omega) \\
& =\int_{U} \mathrm{~d}\left(f^{*} \omega\right) \\
& =\int_{\partial U} f^{*} \omega .
\end{aligned}
$$

We also recall that the space $B V\left(\mathbb{R}^{n}\right)$ is defined as the set of $g \in L^{1}\left(\mathbb{R}^{n}\right)$ such that the total variation $|D g|\left(\mathbb{R}^{n}\right)$ is finite,

$$
|D g|\left(\mathbb{R}^{n}\right)=\sup \left\{\int_{\mathbb{R}^{n}} g \operatorname{div} h \mathrm{~d} x: h \in C^{1}\left(\mathbb{R}^{n} ; \mathbb{R}^{n}\right),|h(x)| \leq 1 \text { for all } x \in \mathbb{R}^{n}\right\} .
$$

Let $C^{1}\left(\mathbb{R}^{n} ; \wedge^{n-1}\right)$ denote the set of $n-1$ forms on $\mathbb{R}^{n}$ with coefficients in $C^{1}\left(\mathbb{R}^{n}\right)$,

$$
\begin{aligned}
C^{1}\left(\mathbb{R}^{n} ; \wedge^{n-1}\right)= & \left\{\sum_{i_{1}<\cdots<i_{n-1}} \omega_{i_{1} \ldots i_{n-1}} \mathrm{~d} x_{i_{1}} \wedge \cdots \wedge \mathrm{d} x_{i_{n-1}}:\right. \\
& \left.\omega_{i_{1} \ldots i_{n-1}} \in C^{1}\left(\mathbb{R}^{n}\right) \text { for all } i_{1}, \ldots, i_{n-1} \in\{1, \ldots, n\}\right\} .
\end{aligned}
$$

Note that the space of $n-1$ forms at a given point $x$ is canonically isomorphic to $\mathbb{R}^{n}$, via

$$
\mathrm{d} x_{\sigma(1)} \wedge \cdots \wedge \mathrm{d} x_{\sigma(n-1)} \mapsto \operatorname{sgn} \sigma e_{\sigma(n)},
$$

for any permutation $\sigma:\{1, \ldots, n\} \rightarrow\{1, \ldots, n\}$. When we write $|\omega(x)|$, we mean the norm of the $n-1$ form $\omega(x)$ under that isomorphism.

By (9) and (10), we have

$$
\begin{aligned}
|D(\operatorname{deg}(\cdot, U, f))|\left(\mathbb{R}^{n}\right) & =\sup \left\{\int_{\mathbb{R}^{n}} \operatorname{deg}(\cdot, U, f) \operatorname{div} h \mathrm{~d} x: h \in C^{1}\left(\mathbb{R}^{n} ; \mathbb{R}^{n}\right),|h| \leq 1\right\} \\
& =\sup \left\{\int_{\partial U} f^{*} \omega: \omega \in C^{1}\left(\mathbb{R}^{n} ; \wedge^{n-1}\right),|\omega| \leq 1\right\} \\
& =\int_{\partial U}\left|D f^{\|}\right| \mathrm{d} \mathcal{H}^{n-1},
\end{aligned}
$$


where $D f^{\|}(x)$ denotes the restriction of the gradient of $D f$ to the space tangent to $\partial U$ (which exists $\mathcal{H}^{n-1}$ almost everywhere by assumption). Finally, recall the Sobolev inequality for $B V$ functions on $\mathbb{R}^{n}$ (see e.g. [22], Theorem 2.1),

$$
\left(\int_{\mathbb{R}^{n}}|f|^{n /(n-1)} \mathrm{d} x\right)^{(n-1) / n} \leq C(n)|D f|\left(\mathbb{R}^{n}\right),
$$

where $C(n)=\left(n \omega_{n}^{1 / n}\right)^{-1}$ is the isoperimetric constant in $\mathbb{R}^{n}$.

Lemma 1. For $v \in C^{2}\left(\overline{B_{1}}\right)$ and $0 \leq r \leq 1$,

$$
\int_{\partial B_{r}}\left|D^{2} v\right| \mathrm{d} \mathcal{H}^{1} \geq\left(4 \pi\left|\int_{B_{r}} \operatorname{det} D^{2} v \mathrm{~d} x\right|\right)^{1 / 2} .
$$

Proof. We write $w:=D v: B_{1} \rightarrow \mathbb{R}^{2}$. By approximation with smooth functions, we may assume $w \in C^{\infty}\left(B_{1} ; \mathbb{R}^{2}\right)$. By (11), we have

$$
\left|D\left(\operatorname{deg}\left(\cdot, B_{r}, w\right)\right)\right|\left(\mathbb{R}^{2}\right) \leq \int_{\partial B_{r}}|D w| \mathrm{d} \mathcal{H}^{1}
$$

By $(12)$, we have

$$
\begin{aligned}
\left|D\left(\operatorname{deg}\left(\cdot, B_{r}, w\right)\right)\right|\left(\mathbb{R}^{2}\right) & \geq\left(4 \pi \int_{\mathbb{R}^{2}}\left|\operatorname{deg}\left(y, B_{r}, w\right) \mathrm{d} y\right|^{2}\right)^{1 / 2} \\
& \geq\left(4 \pi\left|\int_{\mathbb{R}^{2}} \operatorname{deg}\left(y, B_{r}, w\right) \mathrm{d} y\right|\right)^{1 / 2} \\
& =\left(4 \pi\left|\int_{B_{r}} \operatorname{det} D w \mathrm{~d} x\right|\right)^{1 / 2}
\end{aligned}
$$

where we used the fact $\operatorname{deg}\left(x, B_{r}, w\right) \in \mathbb{Z}$, and hence $\left|\operatorname{deg}\left(x, B_{r}, w\right)\right|^{2} \geq\left|\operatorname{deg}\left(x, B_{r}, w\right)\right|$ for almost every $x$, in the second inequality. Combining (13) and (14) yields the claim of the lemma.

\section{Proof of Theorem 1}

Lemma 2. We have

$$
\inf _{y \in W^{2,2}\left(B_{1} ; \mathbb{R}^{3}\right)} I_{h, \Delta}(y) \leq 2 \pi \Delta^{2} h^{2}(|\log h|+C) .
$$

Proof. Recall the definition of $y^{\Delta}$,

$$
y^{\Delta}(x)=\sqrt{1-\Delta^{2}} x+\Delta|x| e_{3} .
$$

Let $\eta \in C^{\infty}([0, \infty))$ with $\eta(t)=0$ for $t \leq \frac{1}{2}, \eta(t)=1$ for $t \geq 1$ and $\left|\eta^{\prime}\right| \leq 4,\left|\eta^{\prime \prime}\right| \leq 8$. We set

$$
y_{0}(x)=\underset{8}{\eta(|x| / h)} y^{\Delta}(x)
$$


We compute

$$
\begin{aligned}
D y_{0}(x)= & \frac{\eta^{\prime}(x / h)}{h} y^{\Delta} \otimes \hat{x}+\eta(x / h) \sqrt{1-\Delta^{2}}\left(\begin{array}{ccc}
1 & 0 & 0 \\
0 & 1 & 0
\end{array}\right)^{T}+\Delta e_{3} \otimes \hat{x} \\
D^{2} y_{0}(x)= & \frac{\eta^{\prime \prime}(x / h)}{h^{2}} y^{\Delta} \otimes \hat{x} \otimes \hat{x}+\frac{\eta^{\prime}(x / h)}{h}\left[\sqrt { 1 - \Delta ^ { 2 } } \left(2 \hat{x} \otimes \hat{x} \otimes \hat{x}+\hat{x}^{\perp} \otimes \hat{x} \otimes \hat{x}^{\perp}\right.\right. \\
& \left.\left.+\hat{x}^{\perp} \otimes \hat{x}^{\perp} \otimes \hat{x}\right)+2 \Delta e_{3} \otimes \hat{x} \otimes \hat{x}+\frac{1}{|x|} y^{\Delta} \otimes \hat{x}^{\perp} \otimes \hat{x}^{\perp}\right] \\
& +\frac{\eta(x / h)}{|x|} \Delta e_{3} \otimes \hat{x}^{\perp} \otimes \hat{x}^{\perp} .
\end{aligned}
$$

On $B_{1} \backslash B_{h}$, we have $y_{0}=y^{\Delta}, \eta^{\prime}(|\cdot| / h)=0$, and hence $D y_{0}^{T} D y_{0}=\left(D y^{\Delta}\right)^{T} D y^{\Delta}$ and $D^{2} y_{0}=|x|^{-1} \Delta e_{3} \otimes \hat{x}^{\perp} \otimes \hat{x}^{\perp}$. On $B_{h}$, we see from the formulas above that $\left|D y_{0}\right| \leq C$ and $\left|D^{2} y_{0}\right| \leq C h^{-1}$. (To see the latter, one uses $\left|y^{\Delta}\right| \leq h$ on $B_{h}$.) Hence the energy can be estimated by

$$
\begin{aligned}
I_{h}\left(y_{0}\right) & \left.\leq \int_{B_{h}}\left(C+h^{2}\left(C h^{-1}\right)^{2}\right)\right) \mathrm{d} x+h^{2} \int_{B_{1} \backslash B_{h}}|x|^{-2} \Delta^{2} \mathrm{~d} x \\
& \leq 2 \pi \Delta^{2} h^{2}(|\log h|+C) .
\end{aligned}
$$

This proves the lemma.

For the following definition, recall the formulation of the Hessian in its very weak form, equation (3).

Definition 1. Let $i \in\{1,2,3\}$. By $\operatorname{det} D^{2} y_{i}^{\Delta}$, we denote the distribution

$$
\varphi \mapsto-\frac{1}{2} \int_{B_{1}} \mathrm{~d} \varphi \wedge\left(y_{i, 1}^{\Delta} \mathrm{d} \hat{y}_{i, 2}-y_{i, 1}^{\Delta} \mathrm{d} y_{i, 2}^{\Delta}\right)
$$

Lemma 3. The distributions $\operatorname{det} D^{2} y_{i}^{\Delta}, i=1,2,3$, can be extended to Radon measures. Denoting the latter with the same symbol, we have

$$
\begin{aligned}
\operatorname{det} D^{2} y_{i}^{\Delta} & =0 \quad \text { for } i=1,2 \\
\operatorname{det} D^{2} y_{3}^{\Delta} & =\Delta^{2} \pi \delta_{0},
\end{aligned}
$$

where $\delta_{0}$ is the Dirac distribution $\delta_{0}: \varphi \mapsto \varphi(0)$. Furthermore, we have

$$
\operatorname{det} D^{2} y_{i}^{\Delta}=\left(y_{i, 1}^{\Delta} \cdot y_{i, 2}^{\Delta}\right)_{, 12}-\frac{1}{2}\left(\left|y_{i, 1}^{\Delta}\right|^{2}\right)_{, 22}-\frac{1}{2}\left(\left|y_{i, 2}^{\Delta}\right|^{2}\right)_{, 11}
$$

in the sense of distributions. 
Proof. For $i=1,2$, the form $y_{i, 1}^{\Delta} \mathrm{d} y_{i, 2}^{\Delta}-y_{i, 1}^{\Delta} \mathrm{d} y_{i, 2}^{\Delta}$ vanishes almost everywhere. Furthermore, we compute

$$
\begin{aligned}
y_{3,1}^{\Delta} \mathrm{d} \hat{y}_{, 2}^{3}-y_{3,1}^{\Delta} \mathrm{d} \hat{y}_{, 2}^{3}= & \Delta^{2}\left[\frac{x_{1}}{|x|}\left(-\frac{x_{1} x_{2}}{|x|^{3}} \mathrm{~d} x_{1}+\frac{x_{1}^{2}}{|x|^{3}} \mathrm{~d} x_{2}\right)\right. \\
& \left.-\frac{x_{2}}{|x|}\left(-\frac{x_{1} x_{2}}{|x|^{3}} \mathrm{~d} x_{2}+\frac{x_{2}^{2}}{|x|^{3}} \mathrm{~d} x_{1}\right)\right] \\
= & \Delta^{2}\left[\frac{x_{1}}{|x|^{2}} \mathrm{~d} x_{2}-\frac{x_{2}}{|x|^{2}} \mathrm{~d} x_{1}\right] .
\end{aligned}
$$

It is well known that the differential of the right hand side is just $-\Delta^{2} 2 \pi \delta_{0} \mathrm{~d} x_{1} \wedge \mathrm{d} x_{2}$. This proves the first claim of the lemma. The claim 15 follows from a simple integration by parts,

$$
\begin{aligned}
& \left\langle\left(y_{i, 1}^{\Delta} y_{i, 2}^{\Delta}\right)_{, 12}-\frac{1}{2}\left(\left|y_{i, 1}^{\Delta}\right|^{2}\right)_{, 22}-\frac{1}{2}\left(\left|y_{i, 2}^{\Delta}\right|^{2}\right)_{, 11}, \varphi\right\rangle \\
& \quad=\frac{1}{2} \int_{B_{1}}\left(\varphi_{, 1}\left(\left(y_{i, 1}^{\Delta} y_{i, 2}^{\Delta}\right)_{, 2}-\left(\left|y_{i, 2}^{\Delta}\right|^{2}\right)_{, 1}\right)+\varphi_{, 2}\left(\left(y_{i, 1}^{\Delta} y_{i, 2}^{\Delta}\right)_{, 1}-\left(\left|y_{i, 1}^{\Delta}\right|^{2}\right)_{, 2}\right)\right) \mathrm{d} x \\
& \quad=\left\langle\operatorname{det} D^{2} y_{i}^{\Delta}, \varphi\right\rangle .
\end{aligned}
$$

This completes the proof of the lemma.

For a given deformation $y \in C^{2}\left(B_{1} ; \mathbb{R}^{3}\right)$, let $\kappa_{y}$ be defined by

$$
\begin{aligned}
\kappa_{y}(\rho) & =\left\langle\sum_{i=1}^{3} \operatorname{det} D^{2} y_{i}, \chi_{B_{\rho}}\right\rangle \\
& =\int_{B_{\rho}} \sum_{i=1}^{3} \operatorname{det} D^{2} y_{i} \mathrm{~d} x \\
& =\sum_{i=1}^{3} \frac{1}{2} \int_{\partial B_{\rho}} y_{i, 1} \mathrm{~d} y_{i, 2}-y_{i, 1} \mathrm{~d} y_{i, 2} .
\end{aligned}
$$

Proposition 1. Let $y \in C^{2}\left(B_{1} ; \mathbb{R}^{3}\right)$ with

Then

$$
\int_{B_{1-5 h} \backslash B_{5 h}}\left|g_{y}-g_{\Delta}\right|^{2}+h^{2}\left|D^{2} y\right|^{2} \mathrm{~d} \mathcal{L}^{2} \leq C h^{2}|\log h|
$$

$$
\left\|\kappa_{y}-\pi \Delta^{2}\right\|_{L^{1}(6 h, R)} \leq C h^{1 / 2} R^{1 / 2}|\log h|^{3 / 4} \quad \text { for all } R \in[2 h, 1-5 h] .
$$

Remark 1. We could prove Theorem 1 via Propositions 1 and 2 also if we made the stronger assumption $I_{h, \Delta}(y) \leq C_{1} h^{2}|\log h|$ in Proposition 1 . However, the weaker assumption will be convenient in the proof of the result on three-dimensional elasticity, Theorem Q.

Proof. Let $h_{0}=h_{0}(y) \in[5 h, 6 h]$ be chosen such that

$$
\int_{\partial B_{h_{0}}}\left|g_{y}-g_{\Delta}\right|^{2} \mathrm{~d} \mathcal{H}^{1}=\int_{\partial B_{h_{0}}}\left|D y^{T} D y-\left(D y^{\Delta}\right)^{T} D y^{\Delta}\right|^{2} \mathrm{~d} \mathcal{H}^{1} \leq C h|\log h| .
$$


For $r \in\left[h_{0}, 1\right]$, we set

$$
\begin{aligned}
F_{1}(r) & =\int_{h_{0}}^{r} \kappa_{y}(\rho) \mathrm{d} \rho, \\
F_{2}(r) & =\left(r-h_{0}\right) \pi \Delta^{2}, \\
F & =F_{1}-F_{2} .
\end{aligned}
$$

By these definitions,

$$
F(r)=\int_{h_{0}}^{r} \sum_{i=1}^{3}\left\langle\operatorname{det} D^{2} y_{i}-\operatorname{det} D^{2} y_{i}^{\Delta}, \chi_{B_{\rho}}\right\rangle \mathrm{d} \rho .
$$

Now we set

$$
u_{i j}=\left(D y^{T} D y-\left(D y^{\Delta}\right)^{T} D y^{\Delta}\right)_{i j} .
$$

By the assumption $y \in C^{2}\left(B_{1} ; \mathbb{R}^{3}\right)$ and Lemma 3 , we have

$$
F(r)=\int_{h_{0}}^{r} \mathrm{~d} \rho \int_{B_{\rho}}\left[u_{12,12}-\frac{1}{2} u_{11,22}-\frac{1}{2} u_{22,11}\right] \mathrm{d} \mathcal{L}^{2} .
$$

Let $k, l \in\{1,2\}$, and $f \in\left\{u_{11}, u_{12}, u_{22}\right\}$. We repeatedly apply Gauss' Theorem,

$$
\begin{aligned}
\int_{h_{0}}^{r} \mathrm{~d} \rho \int_{B_{\rho}} f_{, k l}(x) \mathrm{d} x & =\int_{h_{0}}^{r} \mathrm{~d} \rho \int_{\partial B_{\rho}} \frac{x_{l}}{|x|} f_{, k}(x) \mathrm{d} \mathcal{H}^{1}(x) \\
& =\int_{B_{r} \backslash B_{h_{0}}} \frac{x_{l}}{|x|} f_{, k}(x) \mathrm{d} x \\
& =\int_{\partial\left(B_{r} \backslash B_{h_{0}}\right)} \frac{x_{k}}{|x|} \frac{x_{l}}{|x|} f(x) \mathrm{d} \mathcal{H}^{1}(x)-\int_{B_{r} \backslash B_{h_{0}}}\left(\frac{x_{l}}{|x|}\right)_{, k} f(x) \mathrm{d} x
\end{aligned}
$$

We will estimate the $L^{1}$ norm of the function $G: r \mapsto \int_{h_{0}}^{r} \mathrm{~d} \rho \int_{B_{\rho}} f_{, k l}(x) \mathrm{d} x$. By the triangle inequality and (18), we have

$$
\begin{aligned}
\|G\|_{L^{1}\left(h_{0}, R\right)} \leq & \int_{h_{0}}^{R}\left|\int_{\partial B_{r}} \frac{x_{k}}{|x|} \frac{x_{l}}{|x|} f(x) \mathrm{d} \mathcal{H}^{1}(x)\right| \mathrm{d} r \\
& +\int_{h_{0}}^{R}\left|\int_{\partial B_{h_{0}}} \frac{x_{k}}{|x|} \frac{x_{l}}{|x|} f(x) \mathrm{d} \mathcal{H}^{1}(x)\right| \mathrm{d} r \\
& +\int_{h_{0}}^{R}\left|\int_{B_{r} \backslash B_{h_{0}}}\left(\frac{x_{l}}{|x|}\right)_{, k} f(x) \mathrm{d} x\right| \mathrm{d} r
\end{aligned}
$$

We estimate the terms appearing on the right hand side using the Cauchy-Schwarz inequality. For the first one, we have

$$
\begin{aligned}
\int_{h_{0}}^{R}\left|\int_{\partial B_{r}} \frac{x_{k}}{|x|} \frac{x_{l}}{|x|} f(x) \mathrm{d} \mathcal{H}^{1}(x)\right| \mathrm{d} r & \leq \int_{B_{R} \backslash B_{h_{0}}}\left|\frac{x_{k}}{|x|} \frac{x_{l}}{|x|} f(x)\right| \mathrm{d} \mathcal{L}^{2}(x) \\
\leq & \left(\int_{B_{R} \backslash B_{h_{0}}}|f|^{2} \mathrm{~d} \mathcal{L}^{2}\right)^{1 / 2}\left(\pi\left(R^{2}-h_{0}^{2}\right)^{1 / 2}\right.
\end{aligned}
$$


Secondly, we have

$$
\begin{aligned}
\int_{h_{0}}^{R}\left|\int_{\partial B_{h_{0}}} \frac{x_{k}}{|x|} \frac{x_{l}}{|x|} f(x) \mathrm{d} \mathcal{H}^{1}(x)\right| \mathrm{d} r & =\left(R-h_{0}\right)\left|\int_{\partial B_{h_{0}}} \frac{x_{k}}{|x|} \frac{x_{l}}{|x|} f(x) \mathrm{d} \mathcal{H}^{1}(x)\right| \\
& \leq\left(R-h_{0}\right)\left(\int_{\partial B_{h_{0}}}|f|^{2} \mathrm{~d} \mathcal{H}^{1}\right)^{1 / 2}\left(2 \pi h_{0}\right)^{1 / 2}
\end{aligned}
$$

For the third term,

$$
\begin{aligned}
\int_{h_{0}}^{R}\left|\int_{B_{r} \backslash B_{h_{0}}}\left(\frac{x_{l}}{|x|}\right)_{, k} f(x) \mathrm{d} x\right| \mathrm{d} r & \leq\left(R-h_{0}\right) \int_{B_{R} \backslash B_{h_{0}}} \frac{|f(x)|}{|x|} \mathrm{d} \mathcal{L}^{2}(x) \\
& \leq\left(R-h_{0}\right)\left(\int_{B_{R} \backslash B_{h_{0}}}|f|^{2} \mathrm{~d} \mathcal{L}^{2}\right)^{1 / 2}\left(\int_{B_{R} \backslash B_{h_{0}}}|x|^{-2} \mathrm{~d} \mathcal{L}^{2}(x)\right)^{1 / 2} \\
& \leq\left(R-h_{0}\right)\left(\int_{B_{R} \backslash B_{h_{0}}}|f|^{2} \mathrm{~d} \mathcal{L}^{2}\right)^{1 / 2}\left|\log h_{0}\right|^{1 / 2}
\end{aligned}
$$

Hence, writing $|u|:=\left|D y^{T} D y-\left(D y^{\Delta}\right)^{T} D y^{\Delta}\right|$, we get

$$
\begin{aligned}
\int_{h_{0}}^{R}|F(\rho)| \mathrm{d} \rho \leq & \left(R-h_{0}\right)\left[\left(\int_{\partial B_{h_{0}}}|u|^{2} \mathrm{~d} \mathcal{H}^{1}\right)^{1 / 2}\left(2 \pi h_{0}\right)^{1 / 2}\right. \\
& \left.+\left(\int_{B_{R} \backslash B_{h_{0}}}|u|^{2} \mathrm{~d} \mathcal{L}^{2}\right)^{1 / 2}\left(\int_{B_{R} \backslash B_{h_{0}}}|x|^{-2} \mathrm{~d} \mathcal{L}^{2}\right)^{1 / 2}\right] \\
& +\left(\int_{B_{R} \backslash B_{h_{0}}}|u|^{2} \mathrm{~d} \mathcal{L}^{2}\right)^{1 / 2}\left(\pi\left(R^{2}-h_{0}^{2}\right)\right)^{1 / 2} \\
\leq & C h R|\log h|^{1 / 2} .
\end{aligned}
$$

Furthermore, we have the estimate

$$
\begin{aligned}
\int_{h_{0}}^{R}\left|F^{\prime \prime}(\rho)\right| \mathrm{d} \rho & =\int_{B_{R} \backslash B_{h_{0}}}\left|\sum_{i=1}^{3} \operatorname{det} D^{2} y_{i}\right| \mathrm{d} x \\
& \leq \int_{B_{1-5 h} \backslash B_{5 h}}\left|D^{2} y\right|^{2} \mathrm{~d} x \\
& \leq C|\log h| .
\end{aligned}
$$

Now the claim of the proposition follows from the standard interpolation inequality

$$
\left\|F^{\prime}\right\|_{L^{1}\left(h_{0}, R\right)} \leq C\|F\|_{L^{1}\left(h_{0}, R\right)}^{1 / 2}\left\|F^{\prime \prime}\right\|_{L^{1}\left(h_{0}, R\right)}^{1 / 2},
$$

see e.g. 14], Theorem 7.28. This completes the proof.

Proposition 2. Let $y_{i} \in C^{2}\left(B_{1}\right)$ for $i=1,2,3$. Assume that $\alpha>0$ and

$$
\left\|\kappa_{y}-\pi \Delta^{2}\right\|_{L^{1}(6 h, R)} \leq C h^{1 / 2} R^{1 / 2}|\log h|^{\alpha} \quad \text { for all } R \in[6 h, 1-5 h] .
$$


Then

$$
\sum_{i=1}^{3} \int_{B_{1-5 h} \backslash B_{5 h}}\left|D^{2} y_{i}\right|^{2} \mathrm{~d} x \geq 2 \pi \Delta^{2}(|\log h|-2 \alpha \log |\log h|-C) .
$$

Proof. We set

$$
h_{0}=5 h|\log h|^{2 \alpha}
$$

and choose $J \in \mathbb{N}$ such that

$$
2^{J} h_{0} \leq 1-5 h \leq 2^{J+1} h_{0} .
$$

Note that this choice implies $J \log 2-C \leq\left|\log h_{0}\right| \leq(J+1) \log 2+C$. For $j=0, \ldots, J+1$, we will write $R_{j}:=2^{j} h_{0}$.

We use Jensen's inequality to get for $r \in[5 h, 1-5 h]$,

$$
\int_{\partial B_{r}}\left|D^{2} y_{i}\right|^{2} \mathrm{~d} \mathcal{H}^{1}(x) \geq 2 \pi r\left(\frac{\int_{\partial B_{r}}\left|D^{2} y_{i}\right| \mathrm{d} \mathcal{H}^{1}}{2 \pi r}\right)^{2} \quad \text { for } i \in\{1,2,3\} .
$$

Recall the definition of $\kappa_{y}$ in (17). We use Lemma 1 on the right hand side in (20), sum over $i$, integrate over $r$, and use the triangle inequality to obtain

$$
\sum_{i=1}^{3} \int_{B_{1-5 h} \backslash B_{5 h}}\left|D^{2} y_{i}\right|^{2} \mathrm{~d} x \geq \frac{1}{2 \pi} \int_{5 h}^{1-5 h} \frac{\mathrm{d} r}{r} 4 \pi\left|\kappa_{y}(r)\right| .
$$

Reducing the domain of integration and using again the triangle inequality, we have

$$
\begin{aligned}
\sum_{i=1}^{3} \int_{B_{1-5 h} \backslash B_{5 h}}\left|D^{2} y_{i}\right|^{2} \mathrm{~d} x \geq & 2 \pi \Delta^{2} \int_{R_{0}}^{R_{J}} \frac{\mathrm{d} r}{r}-2 \int_{R_{0}}^{R_{J}} \frac{\mathrm{d} r}{r}\left|\kappa_{y}(r)-\pi \Delta^{2}\right| \\
= & 2 \pi \Delta^{2}(|\log h|-2 \alpha \log |\log h|-C) \\
& -2 \int_{R_{0}}^{R_{J}} \frac{\mathrm{d} r}{r}\left|\kappa_{y}(r)-\pi \Delta^{2}\right| .
\end{aligned}
$$

Using the assumption (19), we may estimate the last term on the right hand side as an error term,

$$
\begin{aligned}
\int_{R_{0}}^{R_{J}} \frac{\mathrm{d} r}{r}\left|\kappa_{y}(r)-\pi \Delta^{2}\right| & =\sum_{j=1}^{J} \int_{R_{j-1}}^{R_{j}} \frac{\mathrm{d} r}{r}\left|\kappa_{y}(r)-\pi \Delta^{2}\right| \\
& \leq \sum_{j=1}^{J} C R_{j}^{-1}\left\|\kappa_{y}-\pi \Delta^{2}\right\|_{L^{1}\left(h, R^{j}\right)} \\
& \leq \sum_{j=1}^{J} C R_{j}^{-1 / 2} h^{1 / 2}|\log h|^{\alpha} \\
& \leq C
\end{aligned}
$$

where in the second inequality, we have used (46), and the last inequality is just the summation of a geometric series. Inserting this in (21), the proof of the proposition is complete. 
Proof of Theorem 1. First note that the minimum is actually attained by some $y \in W^{2,2}\left(B_{1} ; \mathbb{R}^{3}\right)$, since the functional (1) is coercive and lower semi-continuous. The upper bound holds by Lemma 2. Assume that $y \in W^{2,2}\left(B_{1} ; \mathbb{R}^{3}\right)$ with $I_{h, \Delta}(y) \leq 2 \pi \Delta^{2} h^{2}(|\log h|+C)$. By density of $C^{2}$ in $W^{2,2}$, we may assume $y \in C^{2}\left(B_{1} ; \mathbb{R}^{3}\right)$. By Proposition 1 , the assumption of Proposition 2 holds with $\alpha=\frac{3}{4}$. The lower bound now follows from that of Proposition 2.

\section{Three-Dimensional elasticity - Proof of Theorem 2}

This section is concerned with the transition from two dimensions to three. The threedimensional model is defined by the free elastic energy functional (6). We first consider a special class of deformations. Let $y \in C^{2}\left(B_{1, \Delta} ; \mathbb{R}^{3}\right)$ be an immersion, and let the threedimensional deformation $Y$ be given by the following Kirchhoff-Love ansatz:

$$
Y\left(x^{\prime}, x_{3}\right)=y\left(x^{\prime}\right)+x_{3} \nu_{y} \quad \text { for }\left(x^{\prime}, x_{3}\right) \in B_{1, \Delta} \times[-h / 2, h / 2],
$$

where $\nu_{y}=y_{, 1} \wedge y_{, 2} /\left|y_{, 1} \wedge y_{, 2}\right|$ denotes the unit normal. With the assumption (5) on the stored energy density $W$, and provided that $\|D y\|_{L^{\infty}} \leq C$, it is not difficult to show (cf. the proof of Lemma 7 below) that

$$
f_{[-h / 2, h / 2]} W\left(D Y\left(x^{\prime}, x_{3}\right)\right) \mathrm{d} x_{3} \leq C\left(\operatorname{dist}^{2}\left(D y\left(x^{\prime}\right), O(2,3)\right)+h^{2}\left|D^{2} y\left(x^{\prime}\right)\right|^{2}\right),
$$

and hence

$$
E_{h, \Delta}(Y) \leq C \int_{B_{1, \Delta}} \operatorname{dist}^{2}(D y, O(2,3))+h^{2}\left|D^{2} y\right|^{2} \mathrm{~d} \mathcal{L}^{2} .
$$

Whenever a three-dimensional model and a plate model are related to each other through this estimate for the Kirchhoff-Love ansatz, we say for short that they correspond to each other.

As a matter of fact, it is also possible to obtain lower bounds for three-dimensional models from lower bounds in the corresponding plate models. This is based on the Geometric Rigidity Theorem by Friesecke, James, Müller, which we cite now:

Theorem 4. 13] Let $n \geq 2$, and let $U \subset \mathbb{R}^{n}$ be a bounded connected Lipschitz domain. There exists a constant $C^{*}=C^{*}(U)$ with the following property: For every $u \in W^{1,2}\left(U ; \mathbb{R}^{n}\right)$, there exists $R \in S O(n)$ such that

$$
\|D u-R\|_{L^{2}(U)} \leq C^{*} \int_{U} \operatorname{dist}^{2}(D u, S O(n)) \mathrm{d} x .
$$

The constant $C^{*}$ is invariant under rescalings of $U$.

The deduction of lower bounds in three-dimensional elasticity starting from lower bounds for the corresponding plate models via Theorem 4 has been carried out in [30]. The main strategy of our proof is going to be similar to the one given in that reference. Since the estimates needed here differ in many details from those in [30, we nevertheless include a full proof here for the convenience of the reader.

The idea is as follows: From a given deformation in the three-dimensional model, one constructs a deformation in the two-dimensional one through averaging and smoothing. On small boxes of sidelength comparable to $h$ in the 3-dimensional domain, the deformation gradient is close to $S O(3)$ by Theorem 4 . This implies that the gradient of the 
2-dimensional deformation derived from it is close to $O(2,3)$. In this way, the membrane term of the two-dimensional model can be estimated from above by the elastic energy in the 3 -dimensional one. The bending term can be dealt with in a similar way.

We now recall some notation, and introduce some more.

Recall from Section 1.2 that $\iota_{\Delta}: B_{1, \Delta} \rightarrow B_{1}$ is defined by

$$
\iota_{\Delta}(x)=|x|\left(\cos \frac{\varphi(x)}{\sqrt{1-\Delta^{2}}}, \cos \frac{\varphi(x)}{\sqrt{1-\Delta^{2}}}\right),
$$

where $\varphi(x)$ is the angular coordinate of $x$. To alleviate the notation, we write

$$
\iota(x) \equiv \iota_{\Delta}(x)
$$

for $x \in B_{1, \Delta}$. When convenient, we will use the notation $z:=\iota(x)$.

On $\iota\left(B_{1, \Delta}\right)=B_{1} \backslash\left\{\left(x_{1}, 0\right): x_{1} \leq 0\right\}, \iota$ has a well defined inverse, that we denote by

$$
j: B_{1} \backslash\left\{\left(x_{1}, 0\right): x_{1} \leq 0\right\} \rightarrow B_{1, \Delta} .
$$

For $y \in \mathcal{A}^{2 d}$, let $\tilde{y}: \lambda_{h} \rightarrow \mathbb{R}^{3}$ be defined by $\tilde{y}=y \circ j$.

In some places, it will be convenient to work, instead of $B_{1, \Delta}$, with the smaller domain

$$
\omega_{h, \Delta}:=\left\{x \in B_{1-5 h} \backslash B_{5 h}: 0 \leq \arccos \frac{x_{1}}{|x|}<\sqrt{1-\Delta^{2}} \pi\right\} .
$$

By slight abuse of notation, we will not distinguish between the map $\iota$, defined on $B_{1, \Delta}$, and its restriction to $\omega_{h, \Delta}$. In the same way, we will not distinguish between $j: B_{1} \backslash \mathbb{R}_{-} \rightarrow B_{1, \Delta}$ and its restriction to $\lambda_{h}:=\iota\left(\omega_{h, \Delta}\right)=\left(B_{1-5 h} \backslash B_{5 h}\right) \backslash\left\{\left(x_{1}, 0\right): x_{1} \leq 0\right\}$.

If for two quantities $f, g$, there exists a constant $C=C(\Delta)$ such that

$$
\frac{1}{C} f \leq g \leq C f
$$

we will write $f \simeq g$.

Furthermore, for tensors $a \in \mathbb{R}^{3 \times 2 \times 2}$ and $b \in \mathbb{R}^{2 \times 2}$, let $a:(b, b) \in \mathbb{R}^{3 \times 2 \times 2}$ be defined by

$$
(a:(b, b))_{i j k}:=\sum_{l, m=1}^{2} a_{i l m} b_{l j} b_{m k}
$$

for $i \in\{1,2,3\}, j, k \in\{1,2\}$.

4.1. The corresponding plate theory. To prove Theorem 2, we will first have to prove an energy scaling law for a two-dimensional plate model that slightly differs from (1), namely, the one on the right hand side in $(22)$. The reason why we do not choose to work with the three-dimensional model corresponding to (1) is that we want to apply Theorem 4. and the latter requires a flat reference metric - not only in the three-dimensional model, but in the corresponding two-dimensional one too.

Let

$$
\mathcal{A}^{2 d}:=\left\{y \in W_{\text {loc. }}^{2,2}\left(B_{1, \Delta} ; \mathbb{R}^{3}\right): y \circ \iota^{-1} \in W^{2,2}\left(B_{1} ; \mathbb{R}^{3}\right)\right\}
$$


Our two-dimensional plate model is given by $J_{h, \Delta}: \mathcal{A}^{2 d} \rightarrow \mathbb{R}$,

$$
J_{h, \Delta}(y)=\int_{B_{1, \Delta}}\left|g_{y}-\mathrm{Id}_{2 \times 2}\right|^{2}+h^{2}\left|D^{2} y\right|^{2} \mathrm{~d} \mathcal{L}^{2} .
$$

It will be convenient to define a slight variation of $J_{h, \Delta}$, that consists of a reduction of the domain of integration for the energy density. Let

$$
\tilde{J}_{h, \Delta}(y)=\int_{\omega_{h, \Delta}}\left|g_{y}-\operatorname{Id}_{2 \times 2}\right|^{2}+h^{2}\left|D^{2} y\right|^{2} \mathrm{~d} \mathcal{L}^{2} .
$$

Lemma 4. With $\tilde{y}:=y \circ j$, we have for every $y \in \mathcal{A}^{2 d}$,

$$
\begin{aligned}
\tilde{J}_{h, \Delta}(y) \simeq & \int_{\lambda_{h}}\left|g_{\tilde{y}}(z)-g_{\Delta}(z)\right|^{2} \\
& +\left.\left.h^{2}\left|D^{2} \tilde{y}(z)-\Delta^{2}\right| z\right|^{-1}(D \tilde{y}(z) \cdot \hat{z}) \otimes \hat{z}^{\perp} \otimes \hat{z}^{\perp}\right|^{2} \mathrm{~d} \mathcal{L}^{2}(z), \\
J_{h, \Delta}(y) \simeq & \int_{B_{1}}\left|g_{\tilde{y}}(z)-g_{\Delta}(z)\right|^{2} \\
& +\left.\left.h^{2}\left|D^{2} \tilde{y}(z)-\Delta^{2}\right| z\right|^{-1}(D \tilde{y}(z) \cdot \hat{z}) \otimes \hat{z}^{\perp} \otimes \hat{z}^{\perp}\right|^{2} \mathrm{~d} \mathcal{L}^{2}(z) .
\end{aligned}
$$

Proof. For $x \in \omega_{h, \Delta}$, we compute

$$
\begin{aligned}
\left.D \iota\right|_{x} & =\hat{z} \otimes \hat{x}+\frac{1}{\sqrt{1-\Delta^{2}}} \hat{z}^{\perp} \otimes \hat{x}^{\perp} \\
\left.D^{2} \iota\right|_{x} & =-\frac{\Delta^{2}}{1-\Delta^{2}}|x|^{-1} \hat{z} \otimes \hat{x}^{\perp} \otimes \hat{x}^{\perp} .
\end{aligned}
$$

The first equation implies

$$
\left.D \iota\right|_{x} \simeq \mathrm{Id}_{2 \times 2}
$$

in the sense of positive definite matrices. By the chain rule, $\left.D y\right|_{x}=\left.\left.D \tilde{y}\right|_{\iota(x)} \cdot D \iota\right|_{x}$ for $y \in \mathcal{A}^{2 d}$ and $x \in \omega_{h, \Delta}$. As a consequence of the second equation in (24), we have

$$
\begin{aligned}
\left.D^{2} y\right|_{x} & =\left.D^{2} \tilde{y}\right|_{\iota(x)}:\left(\left.D \iota\right|_{x},\left.D \iota\right|_{x}\right)+\left.\left.D \tilde{y}\right|_{\iota(x)} \cdot D^{2} \iota\right|_{x} \\
& =\left.D^{2} \tilde{y}\right|_{\iota(x)}:\left(\left.D \iota\right|_{x},\left.D \iota\right|_{x}\right)-\frac{\Delta^{2}}{1-\Delta^{2}}|x|^{-1}\left(\left.D \tilde{y}\right|_{\iota(x)} \cdot \hat{z}\right) \otimes \hat{x}^{\perp} \otimes \hat{x}^{\perp} .
\end{aligned}
$$

Hence,

$$
\begin{aligned}
\tilde{J}_{h, \Delta}(y)= & \left.\left.\left.\int_{\omega_{h, \Delta}}|D \iota|_{x}^{T} D \tilde{y}\right|_{\iota(x)} ^{T} D \tilde{y}\right|_{\iota(x)} D \iota\right|_{x}-\left.\operatorname{Id}_{2 \times 2}\right|^{2} \\
& +h^{2}\left|D^{2} \tilde{y}\right|_{\iota(x)}:\left(\left.D \iota\right|_{x},\left.D \iota\right|_{x}\right) \\
& -\left.\frac{\Delta^{2}}{1-\Delta^{2}}|x|^{-1}\left(\left.D \tilde{y}\right|_{\iota(x)} \cdot \hat{z}\right) \otimes \hat{x}^{\perp} \otimes \hat{x}^{\perp}\right|^{2} \mathrm{~d} \mathcal{L}^{2}(x) .
\end{aligned}
$$

By (25), we have for every $a \in \mathbb{R}^{3 \times 2 \times 2}$, and for every $b \in \mathbb{R}^{2 \times 2}$,

$$
\begin{aligned}
&\left|a:\left(\left.D \iota\right|_{x} ^{-1},\left.D \iota\right|_{x} ^{-1}\right)\right|^{2} \simeq|a|^{2} \\
&\left.\left|\left(\left.D \iota\right|_{x} ^{-1}\right)^{T} b D \iota\right|_{x}^{-1}\right|^{2} \simeq|b|^{2} . \\
& 16
\end{aligned}
$$


Using this in 26), we get

$$
\begin{aligned}
\tilde{J}_{h, \Delta}(y) \simeq & \left.\int_{\omega_{h, \Delta}}|D \tilde{y}|_{\iota(x)}^{T} D \tilde{y}\right|_{\iota(x)}-\left.\left.D \iota\right|_{x} ^{-1}\left(\left.D \iota\right|_{x} ^{-1}\right)^{T}\right|^{2} \\
& +h^{2}\left|D^{2} \tilde{y}\right|_{\iota(x)}-\left.\Delta^{2}\left(\left.D \tilde{y}\right|_{\iota(x)} \cdot \hat{z}\right) \otimes \hat{z}^{\perp} \otimes \hat{z}^{\perp}\right|^{2} \mathrm{~d} \mathcal{L}^{2}(x) .
\end{aligned}
$$

Multiplying this relation with a factor $|\operatorname{det} D \iota|_{x} \mid=\left(1-\Delta^{2}\right)^{-1 / 2} \simeq 1$, and using the coarea formula, we obtain the first relation in 23); the second is obtained in exactly the same way.

Lemma 5. There exists $y^{h} \in \mathcal{A}^{2 d}$ with

$$
\begin{aligned}
\left|D y^{h}\right| & \leq C \\
\left|D \nu_{y^{h}}\right| & \leq C\left|D^{2} y^{h}\right| \\
J_{h, \Delta}\left(y^{h}\right) & \leq C h^{2}|\log h| .
\end{aligned}
$$

Proof. Let $\tilde{y}^{h}: B_{1} \rightarrow \mathbb{R}^{3}$ be the test function that we constructed in Lemma 2 ; i.e., for $x \in B_{1}$,

$$
\tilde{y}^{h}(x)=\eta(|x| / h) y^{\Delta}(x)
$$

where $\eta \in C^{\infty}([0, \infty))$ with $\eta(t)=0$ for $t \leq \frac{1}{2}, \eta(t)=1$ for $t \geq 1,\left|\eta^{\prime}\right| \leq 4,\left|\eta^{\prime \prime}\right| \leq 8$; and $y^{\Delta}$ is given by

$$
y^{\Delta}(x)=\sqrt{1-\Delta^{2}} x+\Delta e_{3}|x| .
$$

We set $y^{h}:=\tilde{y}^{h} \circ \iota$. From the explicit formulas for $D \tilde{y}^{h}, D^{2} \tilde{y}^{h}$ in the proof of Lemma 2 and (24), we see that $\left|D y^{h}\right| \leq C, D y^{h}=0$ on $B_{h / 2}$, and $\left|D \nu_{y^{h}}\right| \leq C\left|D^{2} y^{h}(z)\right| \leq C|z|^{-1}$ for $z \in B_{1} \backslash B_{h / 2}$. Furthermore, we have $g_{\tilde{y}^{h}}=g_{\Delta}$ on $B_{1} \backslash B_{h}$ and $\left|g_{\tilde{y}^{h}}\right| \leq C$ on $B_{h}$. Hence, using (23), we may estimate

$$
\begin{aligned}
J_{h, \Delta}\left(y^{h}\right) & \simeq \int_{B_{1}}\left|g_{\tilde{y}^{h}}(z)-g_{\Delta}(z)\right|^{2}+\left.\left.h^{2}\left|D^{2} \tilde{y}^{h}(z)-\Delta^{2}\right| z\right|^{-1}\left(D \tilde{y}^{h}(z) \cdot \hat{z}\right) \otimes \hat{z}^{\perp} \otimes \hat{z}^{\perp}\right|^{2} \mathrm{~d} \mathcal{L}^{2}(z) \\
& \leq \int_{B_{h}} C \mathrm{~d} \mathcal{L}^{2}+h^{2} \int_{B_{1} \backslash B_{h / 2}} C|z|^{-2} \mathrm{~d} \mathcal{L}^{2}(z) \\
& \leq C h^{2}(|\log h|+1) .
\end{aligned}
$$

This proves the lemma.

Proposition 3. For all $h$ small enough, we have

$$
\frac{1}{C} h^{2}|\log h| \leq \inf _{y \in \mathcal{A}^{2 d}} \tilde{J}_{h, \Delta}(y) .
$$


Proof. Assume that $\tilde{J}_{h, \Delta}(y) \leq C h^{2}|\log h|$ (otherwise there is nothing to show). Setting $\tilde{y}:=y \circ j$, we may estimate

$$
\begin{aligned}
\int_{B_{1-5 h} \backslash B_{5 h}}|z|^{-2}|D \tilde{y}(z) \cdot \hat{z}|^{2} \mathrm{~d} \mathcal{L}^{2}(z) & \\
\leq & \int_{B_{1-5 h} \backslash B_{5 h}}|z|^{-2} \mathrm{~d} \mathcal{L}^{2}(z)+\int_{B_{1-5 h} \backslash B_{5 h}}|z|^{-2}\left(|D \tilde{y}(z) \cdot \hat{z}|^{2}-1\right) \mathrm{d} \mathcal{L}^{2}(z) \\
\leq & 2 \pi(|\log h|-C) \\
& +\left(\int_{B_{1-5 h} \backslash B_{5 h}}|z|^{-4} \mathrm{~d} \mathcal{L}^{2}(z)\right)^{1 / 2}\left(\int_{B_{1-5 h} \backslash B_{5 h}}\left|g_{\tilde{y}}-g_{\Delta}\right|^{2} \mathrm{~d} \mathcal{L}^{2}(z)\right)^{1 / 2} \\
\leq & 2 \pi|\log h|+C\left(h^{-2}\right)^{1 / 2}\left(h^{2}|\log h|\right)^{1 / 2} \\
\leq & 2 \pi|\log h|+o(|\log h|),
\end{aligned}
$$

where we have used the Cauchy-Schwarz inequality and Lemma 4 in the second inequality. Again by Lemma 4 and Young's inequality, we have

$$
\begin{aligned}
h^{2} \int_{B_{1-5 h} \backslash B_{5 h}}\left|D^{2} \tilde{y}\right| \mathrm{d} \mathcal{L}^{2} & \leq C h^{2}\left(|\log h|+\int_{B_{1-5 h} \backslash B_{5 h}}|z|^{-2}|D \tilde{y} \cdot \hat{z}|^{2} \mathrm{~d} \mathcal{L}^{2}(z)\right) \\
& \leq C h^{2}|\log h| .
\end{aligned}
$$

Hence, by Proposition 1 the assumption of Proposition 2 is fulfilled for $\tilde{y}$ with $\alpha=\frac{3}{2}$. By Proposition 2,

$$
\int_{B_{1-5 h} \backslash B_{5 h}}|D \tilde{y}|^{2} \geq 2 \pi \Delta^{2}\left(|\log h|-\frac{3}{2} \log |\log h|-C\right) .
$$

By (28), 29) and the triangle inequality, we have

$$
\begin{aligned}
& \left\|D^{2} \tilde{y}-\Delta^{2}|z|^{-1}(D \tilde{y} \cdot \hat{z}) \otimes \hat{z}^{\perp} \otimes \hat{z}^{\perp}\right\|_{L^{2}\left(B_{1-5 h} \backslash B_{5 h}\right)}^{2} \\
& \quad \geq\left(\sqrt{2 \pi \Delta^{2}|\log h|}-\sqrt{2 \pi \Delta^{4}|\log h|}\right)^{2}-o(|\log h|) \\
& \quad=2 \pi \Delta(1-\Delta)|\log h|-o(|\log h|) .
\end{aligned}
$$

Combining this last estimate with Lemma 4 proves the proposition.

4.2. Reduction of domain and covers by boxes. As we explained at the beginning of the present section, the core step in the passage from two to three dimensions is to cover the three-dimensional domain by small boxes, and to approximate the deformation by a Euclidean motion on each of these boxes. In this approach, one needs to take special care of the boundary of the two-dimensional domain. Part of that boundary (namely, $\partial B_{1, \Delta} \cap \partial B_{1}$ ) has already been taken care of by "shrinking" $B_{1, \Delta}$ to $\omega_{h, \Delta}$ in the twodimensional plate theory, as we did in the previous subsection. The present subsection's aim is to control the boxes that are close to the remaining part of the boundary. The proof of this subsection's main statement (Lemma 6 below) is straightforward; however, we will need to introduce a certain amount of auxiliary notation. 
We will be interested in coverings of $\omega_{h, \Delta}$ by small squares of sidelength $h$ or $3 h$. For $a \in \mathbb{R}^{2}$ and $r>0$, let $Q_{a, r}:=a+[-r / 2, r / 2]^{2}$. We define

$$
\begin{aligned}
& \mathcal{Q}_{h}:=\left\{a \in h \mathbb{Z}^{2}: Q_{a, h} \cap \omega_{h, \Delta} \neq \emptyset\right\} \\
& \mathcal{Q}_{h}^{*}:=\left\{a \in \mathcal{Q}_{h}: Q_{a, 3 h} \not \subset B_{1, \Delta}\right\} .
\end{aligned}
$$

Furthermore, let

$$
\tilde{B}_{1, \Delta}:=\left\{x \in B_{1} \backslash\{0\}:\left(1-\sqrt{1-\Delta^{2}}\right) \pi<\arccos \frac{x_{1}}{|x|} \leq \pi\right\} .
$$

Now we will define maps $f_{\Delta}^{( \pm)}: \tilde{B}_{1, \Delta} \rightarrow B_{1, \Delta}$ that translate the cut in the domain in angular direction. Let $\phi_{\Delta}:=\left(1-\sqrt{1-\Delta^{2}}\right) 2 \pi$ and let the rotation $S_{\Delta} \in S O(2)$ be defined by

$$
S_{\Delta}=\left(\begin{array}{cc}
\cos \phi_{\Delta} & -\sin \phi_{\Delta} \\
\sin \phi_{\Delta} & \cos \phi_{\Delta}
\end{array}\right)
$$

Let

$$
v^{ \pm}:=\left(\cos \sqrt{1-\Delta^{2}} \pi, \pm \sin \sqrt{1-\Delta^{2}} \pi\right)
$$

Note that $v^{ \pm}$are chosen such that $\left[0, v^{+}\right) \cup\left[0, v^{-}\right)=\partial \tilde{B}_{1, \Delta} \backslash \partial B_{1}$, where by $[a, b)$, we denote the line segment connecting $a$ with $b$ in $\mathbb{R}^{2}$, including $a$, but excluding $b$. Furthermore, note that $S_{\Delta} v^{-}=v^{+}$.

Now let

$$
\begin{aligned}
& \tilde{B}_{1, \Delta,+}:=\left\{x \in \tilde{B}_{1, \Delta}: x \cdot\left(v^{+}\right)^{\perp} \geq 0\right\} \\
& \tilde{B}_{1, \Delta,-}:=\left\{x \in \tilde{B}_{1, \Delta}: x \cdot\left(v^{-}\right)^{\perp} \leq 0\right\}
\end{aligned}
$$

and define $f_{\Delta, \pm}$ by

$$
\begin{aligned}
& f_{\Delta,+}(x)= \begin{cases}S_{\Delta}^{-1} x & \text { if } x \in \tilde{B}_{1, \Delta,+} \\
x & \text { else }\end{cases} \\
& f_{\Delta,-}(x)= \begin{cases}S_{\Delta} x & \text { if } x \in \tilde{B}_{1, \Delta,-} \\
x & \text { else }\end{cases}
\end{aligned}
$$

For a sketch of $\tilde{B}_{1, \Delta, \pm}$ and $f_{\Delta, \pm}$, see Figure 1 .

Note that for $x \in \tilde{B}_{1, \Delta}$,

$$
f_{\Delta,-}^{-1} \circ f_{\Delta,+}(x)= \begin{cases}S_{\Delta}^{-1} x & \text { if } S_{\Delta}^{-1} x \in \tilde{B}_{1, \Delta} \\ S_{\Delta}^{-2} x & \text { else. }\end{cases}
$$

For $Y \in \mathcal{A}_{h, \Delta}$, we define $Y^{( \pm)}: \tilde{B}_{1, \Delta} \times[-h / 2, h / 2] \rightarrow \mathbb{R}^{3}$ by

$$
Y^{( \pm)}\left(x^{\prime}, x_{3}\right)=Y\left(f_{\Delta, \pm}\left(x^{\prime}\right), x_{3}\right) .
$$

The main statement of this subsection is 


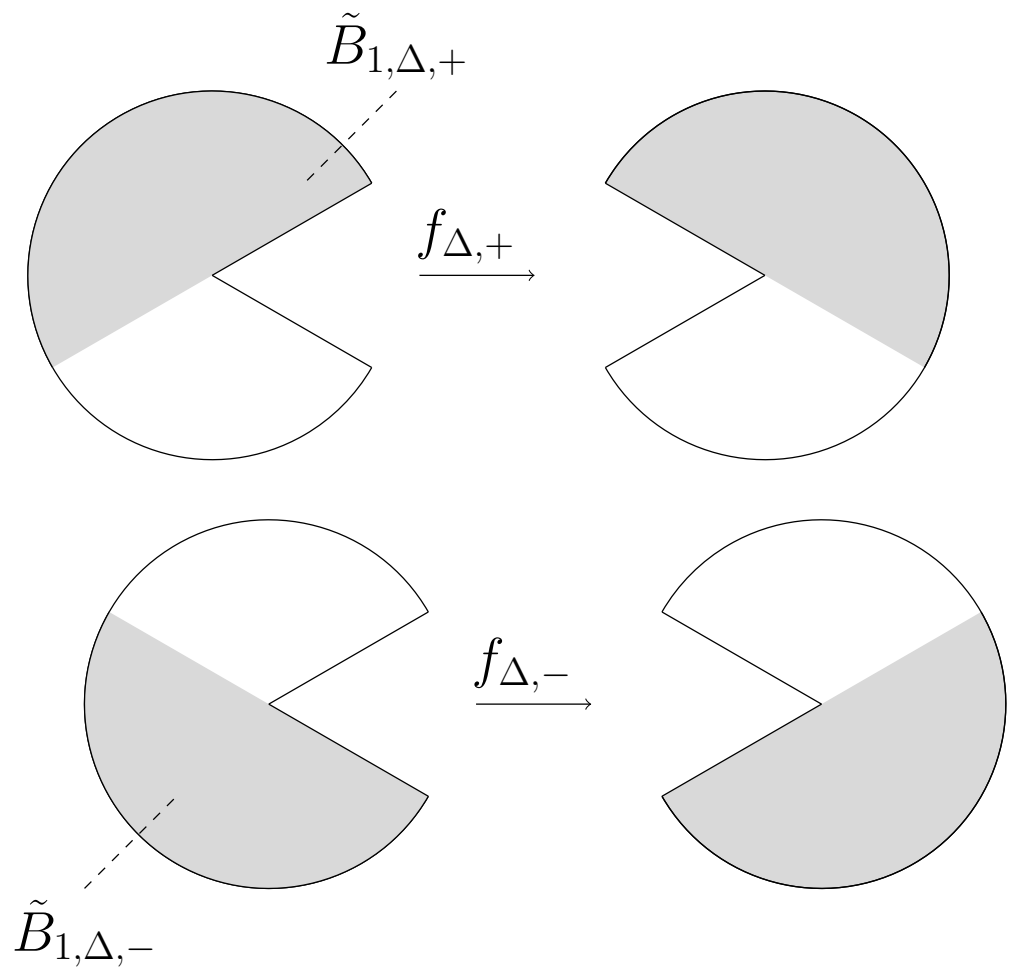

Figure 1. The maps $f_{\Delta, \pm}$. Colored in gray: in the top right, the set $\tilde{B}_{1, \Delta,+}$; in the top right, $f_{\Delta,+}\left(\tilde{B}_{1, \Delta,+}\right)$; in the bottom left, $B_{1, \Delta,-}$; in the bottom right, $f_{\Delta,-}\left(\tilde{B}_{1, \Delta,-}\right)$.

Lemma 6. Let $Y \in \mathcal{A}_{h, \Delta}$. Then

$$
\begin{aligned}
& \sum_{a \in \mathcal{Q}_{h}^{*}} \int_{Q_{a, 3 h} \times[-h / 2, h / 2]} \operatorname{dist}^{2}\left(D Y^{( \pm)}, S O(3)\right) \mathrm{d} \mathcal{L}^{3} \\
& \leq 3 \int_{B_{1, \Delta} \times[-h / 2, h / 2]} \operatorname{dist}^{2}(D Y, S O(3)) \mathrm{d} \mathcal{L}^{3} .
\end{aligned}
$$

Proof. First, we note that as a direct consequence of the definition of $\mathcal{Q}_{h}^{*}$, we have that for every $a \in \mathcal{Q}_{h}^{*}, Q_{a, 3 h} \subset \tilde{B}_{1, \Delta}$. Next, for $g \in L^{1}\left(B_{1, \Delta}\right)$, let

$$
g_{\Delta, \pm}=g \circ f_{\Delta, \pm} \cdot
$$

Note that away from their discontinuity sets (which are $\mathcal{L}^{2}$ null sets), $f_{\Delta, \pm}$ are isometries. Hence, we have

$$
\begin{aligned}
\sum_{a \in \mathcal{Q}_{h}^{*}} \int_{Q_{a, 3 h}}\left|g_{\Delta, \pm}\right| \mathrm{d} \mathcal{L}^{2} & \leq 3 \int_{\tilde{B}_{1, \Delta}}\left|g_{\Delta, \pm}\right| \mathrm{d} \mathcal{L}^{2} \\
& =3 \int_{B_{1, \Delta}}|g| \mathrm{d} \mathcal{L}^{2}
\end{aligned}
$$


For every $x^{\prime} \in \tilde{B}_{1, \Delta}$ that is not on the discontinuity set of $f_{\Delta, \pm}$, there exists $R \in S O(3)$ such that we have

$$
D Y^{( \pm)}\left(x^{\prime}, x_{3}\right)=D Y\left(f_{\Delta, \pm}\left(x^{\prime}\right), x_{3}\right) R
$$

and hence for every $\left(x^{\prime}, x_{3}\right) \in \tilde{B}_{1, \Delta} \times[-h / 2, h / 2]$,

$$
\operatorname{dist}^{2}\left(D Y^{( \pm)}\left(x^{\prime}, x_{3}\right), S O(3)\right)=\operatorname{dist}^{2}\left(D Y\left(f_{\Delta, \pm}\left(x^{\prime}\right), x_{3}\right), S O(3)\right) .
$$

For fixed $x_{3} \in[-h / 2, h / 2]$, combining (34), 35) and the fact that the discontinuity set of $f_{\Delta, \pm}$ is an $\mathcal{L}^{2}$ null set, yields

$\sum_{a \in \mathcal{Q}_{h}^{*}} \int_{Q_{a, 3 h}} \operatorname{dist}^{2}\left(D Y^{( \pm)}\left(x^{\prime}, x_{3}\right), S O(3)\right) \mathrm{d} \mathcal{L}^{2}\left(x^{\prime}\right) \leq 3 \int_{B_{1, \Delta}} \operatorname{dist}^{2}\left(D Y\left(\tilde{x}^{\prime}, x_{3}\right), S O(3)\right) \mathrm{d} \mathcal{L}^{2}\left(\tilde{x}^{\prime}\right)$,

where we have used the coarea formula to make the change of variables $\tilde{x}^{\prime}=f_{\Delta \pm}\left(x^{\prime}\right)$ on the right hand side. Integrating in $x_{3}$ yields the claim of the lemma.

\subsection{Proof of Theorem 2 ,}

Lemma 7. We have

$$
\inf _{Y \in \mathcal{A}_{h, \Delta}} E_{h, \Delta}(Y) \leq C h^{2}|\log h| .
$$

Proof. Let $y^{h}: \omega_{h, \Delta} \rightarrow \mathbb{R}^{3}$ be as in the statement of Lemma 5 . Now, for $\left(x^{\prime}, x_{3}\right) \in$ $B_{1, \Delta} \times[-h / 2, h / 2]$, we set

$$
Y^{h}\left(x^{\prime}, x_{3}\right)=y^{h}\left(x^{\prime}\right)+x_{3} \nu_{y^{h}}\left(x^{\prime}\right) .
$$

We compute

$$
D Y^{h}\left(x^{\prime}, x_{3}\right)=D y^{h}\left(x^{\prime}\right)+\nu_{y^{h}}\left(x^{\prime}\right) \otimes e_{3}+x_{3} D \nu_{y^{h}}\left(x^{\prime}\right) .
$$

By definition of the unit normal $\nu_{y^{h}}$, we have

$$
\operatorname{dist}^{2}\left(D y^{h}\left(x^{\prime}\right)+\nu_{y^{h}}\left(x^{\prime}\right) \otimes e_{3}, S O(3)\right) \leq \operatorname{dist}^{2}\left(D y^{h}\left(x^{\prime}\right), O(2,3)\right) .
$$

By Lemma 5, we have $\left|D \nu_{y^{h}}\right|^{2} \leq C\left|D^{2} y^{h}\right|^{2}$, and hence, we get by integrating in $x_{3}$,

$$
\begin{aligned}
f_{[-h / 2, h / 2]} \operatorname{dist}^{2}( & \left.D Y^{h}\left(x^{\prime}, x_{3}\right), S O(3)\right) \mathrm{d} x_{3} \\
\leq C & \left(\operatorname{dist}^{2}\left(D y^{h}\left(x^{\prime}\right), O(2,3)\right)+h^{2}\left|D^{2} y^{h}\left(x^{\prime}\right)\right|^{2}\right)
\end{aligned}
$$

for all $x^{\prime} \in \omega_{h, \Delta}$.

Since $W(F)<C \operatorname{dist}^{2}(F, S O(3))$ in a neighborhood of $S O(3)$ by (5), we have that for any compact set $\mathcal{F} \subset \mathbb{R}^{3 \times 3}$, there exists a constant $C=C(W, \mathcal{F})$ such that

$$
W(F)<C \operatorname{dist}^{2}(F, S O(3)) \quad \text { for } F \in \mathcal{F} .
$$

Since $\left|D y^{h}\right| \leq C$, we get

$$
\begin{gathered}
W\left(D Y^{h}\right)<C(W) \operatorname{dist}^{2}\left(D y^{h}, S O(3)\right) . \\
21
\end{gathered}
$$


Hence, using (36),

$$
\begin{aligned}
E_{h, \Delta}\left(Y^{h}\right) & =\int_{B_{1, \Delta}} \mathrm{d} x^{\prime} f_{[-h / 2, h / 2]} \mathrm{d} x_{3} W\left(D Y^{h}\right) \\
& \leq C(W) \int_{B_{1, \Delta}} \mathrm{d} x^{\prime}\left(\operatorname{dist}^{2}\left(D y^{h}, O(2,3)\right)+h^{2}\left|D^{2} y^{h}\right|^{2}\right) \\
& \leq C(W) h^{2}|\log h|,
\end{aligned}
$$

where the last estimate holds by Lemma 5 . This proves the lemma.

Proof of Theorem 2. The upper bound has been proved in Lemma 7, while the existence of a minimizer follows from coercivity and lower semicontinuity of $E_{h, \Delta}$ in $W^{1,2}\left(B_{1, \Delta} \times\right.$ $\left.[-h / 2, h / 2] ; \mathbb{R}^{3}\right)$. It remains to show the lower bound.

Step 1. Extension of the domain and mollification. Assume that $Y \in W^{1,2}\left(B_{1, \Delta} \times\right.$ $\left.[-h / 2, h / 2] ; \mathbb{R}^{3}\right)$. We define $y^{(1)}: B_{1, \Delta} \rightarrow \mathbb{R}^{3}$ by setting

$$
y^{(1)}\left(x^{\prime}\right):=f_{[-h / 2, h / 2]} Y\left(x^{\prime}, x_{3}\right) \mathrm{d} x .
$$

For each $x^{\prime} \in \tilde{B}_{1, \Delta}$, we set

$$
y^{(1, \pm)}\left(x^{\prime}\right):=y^{(1)}\left(f_{\Delta, \pm}\left(x^{\prime}\right)\right) .
$$

Next, we mollify; let $\eta \in C_{0}^{\infty}\left(\mathbb{R}^{2}\right)$ with $\int_{\mathbb{R}^{2}} \eta=1$ and $\operatorname{supp} \eta \subset B_{1}$. We set $\eta_{h}(\cdot):=$ $h^{-2} \eta(\cdot / h)$. Now, for $x^{\prime} \in \bigcup_{a \in \mathcal{Q}_{h} \backslash \mathcal{Q}_{h}^{*}} Q_{a, h}$, we set

$$
y^{(2)}\left(x^{\prime}\right)=\left(\eta_{h} * y^{(1)}\right)\left(x^{\prime}\right) .
$$

For $x^{\prime} \in \tilde{B}_{1, \Delta}$, we define

$$
y^{(2, \pm)}\left(x^{\prime}\right)=\left(\eta_{h} * y^{(1, \pm)}\right)\left(x^{\prime}\right)
$$

Since linear transformations and convolution commute, we have by (31),

$$
y^{(2,+)}\left(x^{\prime}\right)=y^{(2,-)}\left(S_{\Delta}^{-1} x^{\prime}\right) \quad \text { for all } x^{\prime} \in \tilde{B}_{1, \Delta} \text { with } S_{\Delta}^{-1} x^{\prime} \in \tilde{B}_{1, \Delta} .
$$

Now let

$$
\omega_{h, \Delta, \pm}=\left\{x \in \bigcup_{a \in \mathcal{Q}_{h}^{*}} Q_{a, h} \cap \omega_{h, \Delta}: f_{\Delta, \pm}\left(x^{\prime}\right)=x^{\prime}\right\} .
$$

Note that we have

$$
\omega_{h, \Delta}=\omega_{h, \Delta,+} \cup \omega_{h, \Delta,-} \cup \bigcup_{a \in \mathcal{Q}_{h} \backslash \mathcal{Q}_{h}^{*}} Q_{a, h},
$$

where the sets on the right hand side are mutually disjoint. On $\omega_{h, \Delta,+} \cup \omega_{h, \Delta,-}$, we define $y^{(2)}$ by

By (37), we have

$$
y^{(2)}\left(x^{\prime}\right)=y^{(2, \pm)}\left(x^{\prime}\right) \quad \text { if } x^{\prime} \in \omega_{h, \Delta, \pm}
$$

$$
y^{(2)} \circ j \in W^{2,2}\left(B_{1-5 h} \backslash B_{5 h} ; \mathbb{R}^{3}\right) .
$$

Step 2. Application of Theorem 4 on small boxes. Let $a \in \mathcal{Q}_{h} \backslash \mathcal{Q}_{h}^{*}$. By Theorem 4 , there exists $R_{a, h} \in S O(3)$ such that

$$
\int_{Q_{a, 3 h} \times[-h / 2, h / 2]} \operatorname{dist}^{2}(D Y, S O(3)) \mathrm{d} x \leq C \int_{Q_{a, 3 h} \times[-h / 2, h / 2]}\left|D Y-R_{a, h}\right|^{2} \mathrm{~d} x .
$$


For $a \in \mathcal{Q}_{h}^{*}$, there exist $R_{a, h}^{( \pm)} \in S O(3)$ such that

$$
\int_{Q_{a, 3 h} \times[-h / 2, h / 2]} \operatorname{dist}^{2}\left(D Y^{( \pm)}, S O(3)\right) \mathrm{d} x \leq C \int_{Q_{a, 3 h} \times[-h / 2, h / 2]}\left|D Y^{( \pm)}-R_{a, h}^{( \pm)}\right|^{2} \mathrm{~d} x
$$

where $Y^{( \pm)}$has been defined in $(32)$. For $a \in \mathcal{Q}_{h} \backslash \mathcal{Q}_{h}^{*}$, let the 3 by 2 matrix consisting of the first two columns of $R_{a, h}$ be denoted by $\hat{R}_{a, h}$. For $a \in \mathcal{Q}_{h}$, let $\hat{R}_{a, h}^{( \pm)}$denote the first two columns of $R_{a, h}^{( \pm)}$respectively.

Step 3. Conclusion. Note that for every $a \in \mathcal{Q}_{h} \backslash \mathcal{Q}_{h}^{*}$, and every $x^{\prime} \in Q_{a, 3 h}$,

$$
\left|D y^{(1)}\left(x^{\prime}\right)-\hat{R}_{a, h}\right| \leq f_{[-h / 2, h / 2]}\left|D Y\left(x^{\prime}, x_{3}\right)-R_{a, h}\right| \mathrm{d} x_{3} .
$$

An analogous formula holds for $a \in \mathcal{Q}_{h}^{*}$. Now we have

$$
\begin{aligned}
\int_{\omega_{h, \Delta}} \operatorname{dist}^{2}\left(D y^{(2)}, O(2,3)\right) \mathrm{d} \mathcal{L}^{2} \leq & \sum_{a \in \mathcal{Q}_{h} \backslash \mathcal{Q}_{h}^{*}} \int_{Q_{a, h}} \operatorname{dist}^{2}\left(D y^{(2)}, O(2,3)\right) \mathrm{d} \mathcal{L}^{2} \\
& +\sum_{a \in \mathcal{Q}_{h}^{*}, i \in\{ \pm\}} \int_{Q_{a, h}} \operatorname{dist}^{2}\left(D y^{(2, i)}, O(2,3)\right) \mathrm{d} \mathcal{L}^{2} \\
\leq & \sum_{a \in \mathcal{Q}_{h} \backslash \mathcal{Q}_{h}^{*}} \int_{Q_{a, h}}\left|D y^{(2)}-\hat{R}_{a, h}\right|^{2} \mathrm{~d} \mathcal{L}^{2} \\
& +\sum_{a \in \mathcal{Q}_{h}^{*}, i \in\{ \pm\}} \int_{Q_{a, h}}\left|D y^{(2, i)}-\hat{R}_{a, h}^{(i)}\right|^{2} \mathrm{~d} \mathcal{L}^{2}
\end{aligned}
$$

We use the definition of $y^{(2)}$ and Young's inequality for convolutions to get (for $a \in \mathcal{Q}_{h} \backslash \mathcal{Q}_{h}^{*}$ )

$$
\begin{aligned}
\int_{Q_{a, h}}\left|D y^{(2)}-\hat{R}_{a, h}\right|^{2} \mathrm{~d} \mathcal{L}^{2} & \leq \int_{Q_{a, h}}\left|\eta_{h} *\left(D y^{(1)}-\hat{R}_{a, h}\right)\right|^{2} \mathrm{~d} \mathcal{L}^{2} \\
& \leq C \int_{Q_{a, 3 h}}\left|D y^{(1)}-\hat{R}_{a, h}\right|^{2} \mathrm{~d} \mathcal{L}^{2}
\end{aligned}
$$

with analogous estimates for $a \in \mathcal{Q}_{h}^{*}$. Inserting in 40, , we get 


$$
\begin{aligned}
\int_{\omega_{h, \Delta}} & \operatorname{dist}^{2}\left(D y^{(2)}, O(2,3)\right) \mathrm{d} \mathcal{L}^{2} \\
\leq & C\left(\sum_{a \in \mathcal{Q}_{h} \backslash \mathcal{Q}_{h}^{*}} \int_{Q_{a, 3 h}}\left|D y^{(1)}-\hat{R}_{a, h}\right|^{2} \mathrm{~d} \mathcal{L}^{2}\right. \\
& \left.+\sum_{a \in \mathcal{Q}_{h}^{*}, i \in\{ \pm\}} \int_{Q_{a, 3 h}}\left|D y^{(1, i)}-\hat{R}_{a, h}^{(i)}\right|^{2} \mathrm{~d} \mathcal{L}^{2}\right) \\
\leq & C h^{-1}\left(\sum_{a \in \mathcal{Q}_{h} \backslash \mathcal{Q}_{h}^{*}} \int_{Q_{a, 3 h} \times[-h / 2, h / 2]}\left|D Y-R_{a, h}\right|^{2} \mathrm{~d} \mathcal{L}^{3}\right. \\
& \left.+\sum_{a \in \mathcal{Q}_{h}^{*}, i \in\{ \pm\}} \int_{Q_{a, 3 h} \times[-h / 2, h / 2]}\left|D Y^{(i)}-R_{a, h}^{(i)}\right|^{2} \mathrm{~d} \mathcal{L}^{3}\right) \\
\leq & C h^{-1}\left(\sum_{a \in \mathcal{Q}_{h} \backslash \mathcal{Q}_{h}^{*}} \int_{Q_{a, 3 h} \times[-h / 2, h / 2]} \operatorname{dist}^{2}(D Y, S O(3)) \mathrm{d} \mathcal{L}^{3}\right. \\
& \left.+\sum_{a \in \mathcal{Q}_{h}^{*}, i \in\{ \pm\}} \int_{Q_{a, 3 h} \times[-h / 2, h / 2]} \operatorname{dist}^{2}\left(D Y^{(i)}, S O(3)\right) \mathrm{d} \mathcal{L}^{3}\right) .
\end{aligned}
$$

Using Lemma 6 in 41, we get

$$
\int_{\omega_{h, \Delta}} \operatorname{dist}^{2}\left(D y^{(2)}, O(2,3)\right) \mathrm{d} \mathcal{L}^{2} \leq C h^{-1} \int_{B_{1, \Delta} \times[-h / 2, h / 2]} \operatorname{dist}^{2}(D Y, S O(3)) \mathrm{d} \mathcal{L}^{3} .
$$

We come to the estimate of the bending term, using again the definition of $y^{(2)}$ and Young's inequality for convolutions:

$$
\begin{aligned}
\int_{\omega_{h, \Delta}}\left|D^{2} y^{(2)}\right|^{2} \mathrm{~d} \mathcal{L}^{2} \leq & \sum_{a \in \mathcal{Q}_{h} \backslash \mathcal{Q}_{h}^{*}} \int_{Q_{a, h}}\left|D \eta_{h} *\left(D y^{(1)}-\hat{R}_{a, h}\right)\right|^{2} \mathrm{~d} \mathcal{L}^{2} \\
& +\sum_{a \in \mathcal{Q}_{h}^{*}, i \in\{ \pm\}} \int_{Q_{a, h}}\left|D \eta_{h} *\left(D y^{(1, i)}-\hat{R}_{a, h}^{(i)}\right)\right|^{2} \mathrm{~d} \mathcal{L}^{2} \\
\leq & C h^{-2}\left(\sum_{a \in \mathcal{Q}_{h} \backslash \mathcal{Q}_{h}^{*}} \int_{Q_{a, 3 h}}\left|D y^{(1)}-\hat{R}_{a, h}\right|^{2} \mathrm{~d} \mathcal{L}^{2}\right. \\
& \left.+\sum_{a \in \mathcal{Q}_{h}^{*}, i \in\{ \pm\}} \int_{Q_{a, 3 h}}\left|D y^{(1, i)}-\hat{R}_{a, h}^{(i)}\right|^{2} \mathrm{~d} \mathcal{L}^{2}\right)
\end{aligned}
$$

Using the chain of inequalities from (41) starting from the second one, and again Lemma 6. we get from 43],

$$
\int_{\omega_{h, \Delta}}\left|D^{2} y^{(2)}\right|^{2} \mathrm{~d} x \leq C h^{-3} \int_{\substack{B_{1, \Delta} \times[-h / 2, h / 2] \\ 24}} \operatorname{dist}^{2}(D Y, S O(3)) \mathrm{d} \mathcal{L}^{3} .
$$


Combining (42) and (44), we get

$$
J_{h, \Delta}\left(y^{(2)}\right) \leq C h^{-1} \int_{B_{1, \Delta} \times[-h / 2, h / 2]} \operatorname{dist}^{2}(D Y, S O(3)) \mathrm{d} \mathcal{L}^{3} .
$$

By (4), this implies

$$
J_{h, \Delta}\left(y^{(2)}\right) \leq C h^{-1} \int_{B_{1, \Delta} \times[-h / 2, h / 2]} W(D Y) \mathrm{d} \mathcal{L}^{3}=C E_{h, \Delta}(Y) .
$$

By Proposition 3, this proves the lower bound, and completes the proof of the theorem.

\section{The Föppl-von Kármán Setting - Proof of Theorem 3}

The Föppl-von Kármán (FvK) plate model is a very popular model for phenomena that include large deflections. Strictly speaking, there exists no rigorous justification for the validity of the model when large deflections occur. The FvK model can be derived from three-dimensional nonlinear elasticity as a low energy Gamma-limit, see [13, 12]. This rigorously justifies the FvK model, but only as a model for small deflections.

Nevertheless, the FvK equations, first formulated more than hundred years ago [32, have a long and quite successful history of describing large deformation phenomena in engineering, from the design of submarine hulls over paper crumpling [31, 1, 19, 21] and wrinkling to the mechanical properties of cell walls [29, 18]. In the context of stability and buckling, several interesting points on why the FvK equations are relevant, even in regimes that they seemingly do not describe, have been made in [17].

In the applications mentioned above, the FvK model usually still contains a small parameter $h$ that is interpreted as the (rescaled) thickness of the sheet, whereas in the Gamma-Limit from [12], the thickness has disappeared from the model. Strictly speaking, it is unclear if plate models that still contain the small parameter $h$ have a rigorous meaning asymptotically.

Theorem 3 identifies the energy scaling of a single disclination in the FvK model that includes the small parameter $h$. The size of the disclination is of the same order as the "natural" deflection size in the FvK model, and is determined by the parameter $\Delta$.

To see the relation between $I_{h, \Delta}$ and $I_{h, \Delta}^{\mathrm{vK}}$, let $\epsilon$ be a small parameter, and set $\tilde{\Delta}=\epsilon \Delta$, $\tilde{h}=\epsilon h$. Assume further that $(u, v) \in C^{\infty}\left(B_{1} ; \mathbb{R}^{2}\right) \times C^{\infty}\left(B_{1}\right)$, and that the deformation $y: B_{1} \rightarrow \mathbb{R}^{3}$ is given by

$$
y(x)=x+\epsilon^{2} u+\epsilon v e_{3} .
$$

Now we have

$$
\begin{aligned}
& D y=e_{1} \otimes e_{1}+e_{2} \otimes e_{2}+\epsilon^{2} D u+\epsilon e_{3} \otimes D v \\
& D y^{T} D y=\operatorname{Id}_{2 \times 2}+\epsilon^{2}\left(D u+D u^{T}+D v \otimes D v\right)+O\left(\epsilon^{3}\right) \\
& D y^{T} D y-g_{\tilde{\Delta}}=\epsilon^{2}\left(D u+D u^{T}+D v \otimes D v+\Delta^{2} \hat{x}^{\perp} \otimes \hat{x}^{\perp}\right) \text {, }
\end{aligned}
$$


and hence

$$
\begin{aligned}
I_{\tilde{h}, \tilde{\Delta}}(y) & =\int_{B_{1}}\left|g_{y}-g_{\tilde{\Delta}}\right|^{2}+\tilde{h}^{2}\left|D^{2} y\right|^{2} \mathrm{~d} \mathcal{L}^{2} \\
& =\epsilon^{4} \int_{B_{1}}\left|2 \operatorname{sym} D u+D v \otimes D v+\Delta^{2} \hat{x}^{\perp} \otimes \hat{x}^{\perp}\right|^{2}+h^{2}\left|D^{2} v\right|^{2} \mathrm{~d} \mathcal{L}^{2}+O\left(\epsilon^{5}\right) .
\end{aligned}
$$

Hence, we see that $I_{h, \Delta}^{\mathrm{vK}}(u, v)$ is the lowest order term in $\epsilon$ of $I_{\epsilon h, \epsilon \Delta}\left(x \mapsto x+\epsilon^{2} u+\epsilon v e_{3}\right)$. We remark that the above expansion in $\epsilon$ can not be used to deduce Theorem 3 from Theorem 1 in a trivial way. Nevertheless, the proof of Theorem 3 is very similar to the one of Theorem 1. The role that was played by the control variable $\kappa_{y}(r)=\int_{B_{r}} \sum_{i} \operatorname{det} D^{2} y_{i} \mathrm{~d} x$ there will be played by

$$
\kappa_{v}^{\mathrm{vK}}(r):=\int_{B_{r}} \operatorname{det} D^{2} v \mathrm{~d} x
$$

here. We recall that $\operatorname{det} D^{2} v$ is the Föppl-von Kármán version of Gauss curvature - so our control variable is a curvature integral, and by Gauss' equation may be thought of as the (oriented) volume of balls $B_{r}$ under the push-forward of the Gauss map.

The only significant difference between the two cases is that we will have to identify the right terms in the membrane energy to make estimates for $\kappa_{v}^{\mathrm{vK}}$ in the $W^{-1,1}$ norm.

We start off with the upper bound:

Lemma 8. We have

$$
\inf _{(u, v) \in \mathcal{A}} I_{h, \Delta}^{\mathrm{vK}}(u, v) \leq 2 \pi \Delta^{2} h^{2}|\log h|+C h^{2} .
$$

Proof. Let $\eta \in C^{\infty}([0, \infty))$ with $\eta(x)=0$ for $x \leq \frac{1}{2}, \eta(x)=1$ for $x \geq 1$ and $\left|\eta^{\prime}\right| \leq 4$, $\left|\eta^{\prime \prime}\right| \leq 8$. Now we define $u^{h}: B_{1} \rightarrow \mathbb{R}^{2}, v^{h}: B_{1} \rightarrow \mathbb{R}$ by

$$
\begin{aligned}
u^{h}(x) & :=-\frac{\Delta^{2}}{2} \eta(|x| / h) x \\
v^{h}(r, \varphi) & :=\Delta \eta(|x| / h)|x| .
\end{aligned}
$$

The membrane energy density $\left|2 \operatorname{sym} D u^{h}+D v^{h} \otimes D v^{h}+\Delta^{2} \hat{x}^{\perp} \otimes \hat{x}^{\perp}\right|^{2}$ vanishes on $B_{1} \backslash$ $B_{h}$, while it is bounded by a constant $C$ that is independent of $h$ on $B_{h}$. Hence

$$
E_{\text {membrane }}:=\int_{B_{1}}\left|2 \operatorname{sym} D u^{h}+D v^{h} \otimes D v^{h}+\Delta^{2} \hat{x}^{\perp} \otimes \hat{x}^{\perp}\right|^{2} \mathrm{~d} x \leq C h^{2} .
$$

Furthermore,

$$
D^{2} v^{h}=\Delta\left(\frac{|x|}{h^{2}} \eta^{\prime \prime}(|x| / h)+\frac{2}{h} \eta^{\prime}(|x| / h)\right) \hat{x} \otimes \hat{x}+\frac{\Delta}{|x|}\left(\frac{|x|}{h} \eta^{\prime}(|x| / h)+\eta(|x| / h)\right) \hat{x}^{\perp} \otimes \hat{x}^{\perp} .
$$

Hence

$$
\begin{aligned}
& \left|D^{2} v^{h}\right|^{2} \leq C h^{-2} \quad \text { for }|x| \leq C h^{-2}, \\
& \left|D^{2} v^{h}\right|^{2}=\Delta^{2}|x|^{-2} \quad \text { for }|x|>h,
\end{aligned}
$$

and

$$
\begin{aligned}
h^{-2} E_{\text {bending }}:=\int_{B_{1}}\left|D^{2} v^{h}\right|^{2} \mathrm{~d} x & \leq \int_{B_{h}} C h^{-2} \mathrm{~d} x+2 \pi \int_{h}^{1} \Delta^{2} \frac{\mathrm{d} r}{r} \\
& \leq 2 \pi \Delta^{2}|\log h|+C .
\end{aligned}
$$


This proves the lemma.

We come to the interpolation between metric and curvature that will yield an $L^{1}$ estimate for $\kappa_{v}^{\mathrm{vK}}$.

Proposition 4. Let $(u, v) \in C^{2}\left(B_{1} ; \mathbb{R}^{3}\right)$ with $I_{h}^{\mathrm{vK}}(y) \leq 2 \pi \Delta^{2} h^{2}(|\log h|+C)$. Then

$$
\left\|\kappa_{v}^{\mathrm{vK}}-\pi \Delta^{2}\right\|_{L^{1}(h, R)} \leq C h^{1 / 2} R^{1 / 2}|\log h| .
$$

Proof. In the present proof, we will use polar coordinates $r, \varphi$ on $B_{1}$. The unit vectors in $r$ and $\varphi$ direction are denoted by $e_{r}=\hat{x}$ and $e_{\varphi}=\hat{x}^{\perp}$ respectively. The vector field $u$ will be written as $u=u_{r} e_{r}+u_{\varphi} e_{\varphi}$. The membrane energy in these coordinates is given by

$$
\begin{aligned}
\int_{0}^{1} r \mathrm{~d} r \int_{0}^{2 \pi} \mathrm{d} \varphi & \left(\left|2 u_{r, r}+v_{, r}^{2}\right|^{2}+\left|2 r^{-1}\left(u_{, \varphi}^{\varphi}+u_{r}\right)+\left(r^{-1} v_{, \varphi}\right)^{2}+\Delta^{2}\right|^{2}\right. \\
& \left.+2\left|u_{\varphi, r}+r^{-1}\left(u_{r, \varphi}-u_{\varphi}+v_{, r} v_{, \varphi}\right)\right|^{2}\right)
\end{aligned}
$$

For $r>0$, we have

$$
\int_{B_{r}} \operatorname{det} D^{2} v \mathrm{~d} x=\frac{1}{2} \int_{\partial B_{r}} v_{, 1} \mathrm{~d} v_{, 2}-v_{, 2} \mathrm{~d} v_{, 1} .
$$

With $v_{, 1}=v_{, r} \cos \varphi-r^{-1} v_{, \varphi} \sin \varphi$ and $v_{, 2}=v_{, r} \sin \varphi+r^{-1} v_{, \varphi} \cos \varphi$, we compute

$$
\begin{aligned}
v_{, 1} \mathrm{~d} v_{, 2}-v_{, 2} \mathrm{~d} v_{, 1} & =\alpha \mathrm{d} r+\left(v_{, r}^{2}+\left(\frac{v_{, \varphi}}{r}\right)^{2}+\frac{v_{, r} v_{, \varphi \varphi}}{r}-\frac{v_{, \varphi} v_{, r \varphi}}{r}\right) \mathrm{d} \varphi \\
& =\alpha \mathrm{d} r+\left(\left(v_{, r}\right)^{2}-\left(\frac{v_{, \varphi}^{2}}{r}\right)_{, r}+\frac{\left(v_{, r} v_{, \varphi}\right)_{, \varphi}}{r}\right) \mathrm{d} \varphi,
\end{aligned}
$$

where $\alpha$ is some function of $r, \varphi$ that will be irrelevant for our purpose. For $h \leq s, r \leq 1$, let

$$
\begin{aligned}
a(s) & :=\int_{h}^{s} \mathrm{~d} r \int_{0}^{2 \pi} \mathrm{d} \varphi\left(2 u_{r, r}+v_{, r}^{2}\right) \\
b(r) & :=\int_{0}^{2 \pi} \mathrm{d} \varphi\left(2 u_{r}+\frac{v_{, \varphi}^{2}}{r}+\Delta^{2} r\right)
\end{aligned}
$$

Now we set $F(s):=\frac{1}{2}(a(s)-b(s))$, and compute

$$
F^{\prime}(r)=\frac{1}{2} \int \mathrm{d} \varphi\left(v_{, r}^{2}-\left(\frac{v_{, \varphi}^{2}}{r}\right)_{, r}-\Delta^{2}\right) .
$$

Comparing this last expression with (47) and (48), we have

$$
\begin{aligned}
& F^{\prime}(r)=-\pi \Delta^{2}+\int_{B_{r}} \operatorname{det} D^{2} v \mathrm{~d} x \\
& F^{\prime \prime}(r)=\int r \mathrm{~d} \varphi \operatorname{det} D^{2} v . \\
& 27
\end{aligned}
$$


This gives us the needed bound on $\left\|F^{\prime \prime}\right\|_{L^{1}}$ :

$$
\begin{aligned}
\left\|F^{\prime \prime}\right\|_{L^{1}(h, 1)} & \leq \int_{0}^{1} r \mathrm{~d} r \int_{0}^{2 \pi} \mathrm{d} \varphi\left|\operatorname{det} D^{2} v\right| \\
& \leq \int_{B_{1}}\left|D^{2} v\right| \mathrm{d} x \\
& \leq C|\log h| .
\end{aligned}
$$

The $L^{1}$ bound on $F$ works as follows,

$$
\begin{aligned}
\int_{h}^{R}|b(r)| \mathrm{d} r & =\int_{h}^{R} \mathrm{~d} r\left|\int \mathrm{d} \varphi\left(2 u_{r}+\frac{v_{, \varphi}^{2}}{r}+\Delta^{2} r\right)\right| \\
& \leq\left(\int_{h}^{1} r \mathrm{~d} r \mathrm{~d} \varphi\left|2 r^{-1}\left(u_{\varphi, \varphi}+u_{r}\right)+\left(r^{-1} v_{, \varphi}\right)^{2}+\Delta^{2}\right|^{2}\right)^{1 / 2}\left(\int_{h}^{R} r \mathrm{~d} r\right)^{1 / 2} \\
& \leq C h|\log h|^{1 / 2} R
\end{aligned}
$$

and

$$
\begin{aligned}
|a(s)|=\left|\int_{h}^{s} \mathrm{~d} r \int \mathrm{d} \varphi\left(2 u_{r, r}+v_{, r}^{2}\right)\right| & \leq\left(\int_{0}^{1} r \mathrm{~d} r \int \mathrm{d} \varphi\left|2 u_{r, r}+v_{, r}^{2}\right|^{2}\right)^{1 / 2}\left(\int_{h}^{1} \frac{\mathrm{d} r}{r}\right)^{1 / 2} \\
& \leq C h|\log h|
\end{aligned}
$$

from which we get

$$
\int_{h}^{R}|a(r)| \mathrm{d} r \leq C h R|\log h|
$$

In conclusion,

$$
\int_{h}^{R}|F(r)| \mathrm{d} r \leq C h R|\log h|
$$

Using the standard interpolation inequality

$$
\left\|F^{\prime}\right\|_{L^{1}(h, R)} \leq C\|F\|_{L^{1}(h, R)}^{1 / 2}\left\|F^{\prime \prime}\right\|_{L^{1}(h, R)}^{1 / 2},
$$

and 49 , we obtain the claim of the proposition.

The proof of Theorem 3 now almost works exactly in the same way as the proof of Theorem 1.

Proof of Theorem 3. The existence of a minimum follows from coercivity and lower semicontinuity of the functional (7). The upper bound follows from Lemma 8. Assume that $(u, v) \in W^{1,2}\left(B_{1} ; \mathbb{R}^{3}\right) \times W^{2,2}\left(B_{1} ; \mathbb{R}^{3}\right)$ with $I_{h, \Delta}^{\mathrm{vK}} \leq 2 \pi \Delta^{2} h^{2}(|\log h|+C)$. By density of $C^{2}\left(B_{1} ; \mathbb{R}^{3}\right)$ in $W^{1,2}\left(B_{1} ; \mathbb{R}^{3}\right) \times W^{2,2}\left(B_{1} ; \mathbb{R}^{3}\right)$, we may assume $(u, v) \in C^{2}\left(B_{1} ; \mathbb{R}^{3}\right)$. By Proposition 4, we may assume that the assumptions of Proposition 2 hold with $\alpha=1$ and

$$
y_{1}=y_{2}=0, \quad y_{3}=v .
$$

With this choice of $y_{i}$, we have $\kappa_{y}=\kappa_{v}^{\mathrm{vK}}$ and

$$
\sum_{i=1}^{3} \int_{B_{1}}\left|D^{2} y_{i}\right|^{2} \mathrm{~d} x=\int_{B_{1}}\left|D^{2} v\right|^{2} \mathrm{~d} x .
$$


Hence, the lower bound follows from the statement of Proposition 2. This completes the proof of the theorem.

\section{REFERENCES}

[1] Basile Audoly and Yves Pomeau. Elasticity and geometry: from hair curls to the non-linear response of shells. Oxford University Press, 2010.

[2] M. Ben Amar and Y. Pomeau. Crumpled paper. Proc. Roy. Soc. London Ser. A, 453(1959):729-755, 1997.

[3] Jeremy Brandman, Robert V. Kohn, and Hoai-Minh Nguyen. Energy scaling laws for conically constrained thin elastic sheets. J. Elasticity, 113(2):251-264, 2013.

[4] D.L.D. Caspar and A. Klug. Physical principles in the construction of regular viruses. In Cold Spring Harbor Symposia on Quantitative Biology, volume 27, pages 1-24. Cold Spring Harbor Laboratory Press, 1962.

[5] E. Cerda, S. Chaieb, F. Melo, and L. Mahadevan. Conical dislocations in crumpling. Nature, 401:4649, 1999.

[6] E. Cerda and L. Mahadevan. Conical surfaces and crescent singularities in crumpled sheets. Phys. Rev. Lett., 80:2358-2361, Mar 1998.

[7] E. Cerda and L. Mahadevan. Confined developable elastic surfaces: cylinders, cones and the elastica. Proceedings of the Royal Society A: Mathematical, Physical and Engineering Science, 461(2055):671700, 2005.

[8] Sergio Conti and Francesco Maggi. Confining thin elastic sheets and folding paper. Arch. Ration. Mech. Anal., 187(1):1-48, 2008.

[9] Julien Dervaux, Pasquale Ciarletta, and Martine Ben Amar. Morphogenesis of thin hyperelastic plates: a constitutive theory of biological growth in the Föppl-von Kármán limit. J. Mech. Phys. Solids, $57(3): 458-471,2009$.

[10] B. A. DiDonna and T. A. Witten. Anomalous strength of membranes with elastic ridges. Phys. Rev. Lett., 87:206105, Oct 2001.

[11] Irene Fonseca and Wilfrid Gangbo. Degree theory in analysis and applications, volume 2 of Oxford Lecture Series in Mathematics and its Applications. The Clarendon Press, Oxford University Press, New York, 1995. Oxford Science Publications.

[12] G. Friesecke, R. D. James, and S. Müller. A hierarchy of plate models derived from nonlinear elasticity by gamma-convergence. Arch. Ration. Mech. Anal., 180(2):183-236, 2006.

[13] Gero Friesecke, Richard D. James, and Stefan Müller. A theorem on geometric rigidity and the derivation of nonlinear plate theory from three-dimensional elasticity. Comm. Pure Appl. Math., 55(11):1461-1506, 2002

[14] D. Gilbarg and N.S. Trudinger. Elliptic partial differential equations of second order. Classics in mathematics. Springer, 2001.

[15] E. M. Kramer and T. A. Witten. Stress condensation in crushed elastic manifolds. Phys. Rev. Lett., 78:1303-1306, Feb 1997.

[16] Nicolaas H. Kuiper. On $C^{1}$-isometric imbeddings. I, II. Nederl. Akad. Wetensch. Proc. Ser. A. 58, Indag. Math., 17:545-556, 683-689, 1955.

[17] Myriam Lecumberry and Stefan Müller. Stability of slender bodies under compression and validity of the von Kármán theory. Arch. Ration. Mech. Anal., 193(2):255-310, 2009.

[18] Jack Lidmar, Leonid Mirny, and David R Nelson. Virus shapes and buckling transitions in spherical shells. Physical Review E, 68(5):051910, 2003.

[19] A. Lobkovsky, S. Gentges, H. Li, D. Morse, and T. A. Witten. Scaling properties of stretching ridges in a crumpled elastic sheet. Science, 270(5241):1482-1485, 1995.

[20] A. E. Lobkovsky. Boundary layer analysis of the ridge singularity in a thin plate. Phys. Rev. E, 53:3750-3759, April 1996.

[21] A. E. Lobkovsky and T. A. Witten. Properties of ridges in elastic membranes. Phys. Rev. E, 55:15771589, February 1997.

[22] Vladimir Maz'ya. Lectures on isoperimetric and isocapacitary inequalities in the theory of Sobolev spaces. In Heat kernels and analysis on manifolds, graphs, and metric spaces (Paris, 2002), volume 338 of Contemp. Math., pages 307-340. Amer. Math. Soc., Providence, RI, 2003. 
[23] Stefan Müller and Heiner Olbermann. Almost conical deformations of thin sheets with rotational symmetry. SIAM J. Math. Anal., 46(1):25-44, 2014.

[24] Stefan Müller and Heiner Olbermann. Conical singularities in thin elastic sheets. Calc. Var. Partial Differential Equations, 49(3-4):1177-1186, 2014.

[25] John Nash. $C^{1}$ isometric imbeddings. Ann. of Math. (2), 60:383-396, 1954.

[26] Heiner Olbermann. Energy scaling law for the regular cone. J. Nonlinear Sci., 2015. Accepted for publication; arXiv preprint arXiv:1502.07013.

[27] A. V. Pogorelov. Extrinsic geometry of convex surfaces. American Mathematical Society, Providence, R.I., 1973. Translated from the Russian by Israel Program for Scientific Translations, Translations of Mathematical Monographs, Vol. 35.

[28] A. E. Romanov. Mechanics and physics of disclinations in solids. Eur. J. Mech. A Solids, 22(5):727741, 2003. 5th EUROMECH Solid Mechanics Conference (Thessaloniki, 2003).

[29] H. S. Seung and David R. Nelson. Defects in flexible membranes with crystalline order. Phys. Rev. A, 38:1005-1018, Jul 1988

[30] Thilo Simon. $\Gamma$-equivalence for nonlinear plate theories. Master's thesis, Universität Bonn, 2014.

[31] S. C. Venkataramani. Lower bounds for the energy in a crumpled elastic sheet - a minimal ridge. Nonlinearity, 17(1):301-312, 2004.

[32] Theodore Von Karman. Festigkeitsprobleme im maschinenbau. publisher not identified, 1910.

[33] T. A. Witten. Stress focusing in elastic sheets. Rev. Mod. Phys., 79:643-675, Apr 2007.

[34] TA Witten and Hao Li. Asymptotic shape of a fullerene ball. EPL (Europhysics Letters), 23(1):51, 1993.

[35] Arash Yavari and Alain Goriely. Riemann-Cartan geometry of nonlinear disclination mechanics. Math. Mech. Solids, 18(1):91-102, 2013.

(Heiner Olbermann) Hausdorff Center for Mathematics, Bonn, Germany

E-mail address: heiner.olbermann@hcm.uni-bonn.de 